\title{
Artigo \\ Utilização de métodos numéricos na resolução de equações diferenciais que descrevem problemas de sistema massa-mola com amortecimento
}

\author{
Modesto Valci Moreira Lopes ${ }^{[1]}$, Matheus da Silva Menezes ${ }^{[2]}$
}

[1] Universidade Federal Rural do Semi-árido; modsval@gmail.com

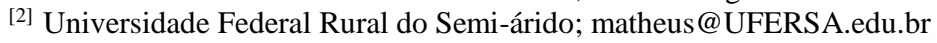

Recebido: 26/06/2019;

Aceito: 21/08/ano;

Publicado: 07/10/2019.

Resumo: Diversos problemas de engenharia podem ter seus comportamentos descritos por equações diferenciais, proporcionando então, uma análise mais teórica dos seus comportamentos e facilitando a compreensão dos mesmos. Diversos métodos analíticos são utilizados na resolução de equações diferenciais, no entanto, nem sempre é possível se obter uma solução analítica, o que nos leva então a utilizar outros modelos matemáticos para tal função, os métodos numéricos. O presente trabalho tem como objetivo avaliar a eficiência da utilização dos métodos numéricos de Euler, Heun e Runge - Kutta, na resolução de equações diferenciais que descrevem problemas de sistema massa-mola com amortecimento viscoso. Para a resolução dos testes, foram realizadas simulações numéricas a partir da implementação dos métodos no IDE SciLab 5.5.2, onde diversas curvas foram obtidas a ponto de propiciarem a avaliação de desempenho de cada método.

Palavras-chave: Sistema massa-mola; equações diferenciais; métodos numéricos; aplicações numéricas.

\section{INTRODUÇÃO}

Segundo [1], muitos problemas fundamentais da mecânica, biologia, física, química, economia e nas

$\mathcal{N}_{\text {diversas áreas da engenharia são dados em termos de variações espaciais e/ou temporais, e definem }}$ mecanismos de variação. A abordagem de tais problemas é feita através de Equações Diferenciais, que envolvem uma função desconhecida e suas respectivas derivadas.

Uma importante aplicação na engenharia é o estudo e análise das vibrações. Em qualquer estrutura civil, mecanismos ou máquinas em geral esse fenômeno pode estar presente, ocasionando, quando ultrapassa os devidos limites, falhas e problemas muitas vezes irreversíveis e até mesmo fatais para quem está utilizando ou operando.

Diante da importância e complexidade na interpretação e resolução dos problemas que envolvem vibrações de sistemas massa-mola com amortecimento, torna-se necessário a utilização e resolução de equações diferenciais.

Para a resolução de equações diferenciais existem diversos métodos analíticos, porém, existem situações [4] em que não é possível obter uma solução analítica, sendo assim, torna-se necessária a utilização de métodos numéricos, modelos matemáticos que a partir de uma função própria se aproxima da solução de um problema. Entre esses métodos vale ressaltar o Método de Euler, o Método de Heun e o Método de Runge-Kutta.

Esse trabalho tem como objetivo analisar a eficiência da utilização dos métodos numéricos de Euler, Heun e Rugen-Kutta na resolução de equações diferenciais que descrevem problemas de sistema massa-mola com amortecimento, em relação às soluções analíticas obtidas na literatura.

\section{FUNDAMENTAÇÃO TEÓRICA}

\subsection{Equações Diferenciais}

Para uma melhor compreensão dos estudos desenvolvidos neste trabalho, será apresentado um breve resumo da teoria fundamental relacionada às equações diferenciais. As demonstrações dos teoremas mais gerais fogem 
do escopo desse trabalho e, portanto, não serão expostas no texto. Para darmos início ao estudo, é necessário que se entenda o que é uma equação diferencial. Entre outros autores, [3] vem mostrar que:

Definição 2.1: Uma equação que contém as derivadas (ou diferenças) de uma ou mais variáveis dependentes em relação a uma ou mais variáveis independentes é chamada de equação diferencial (ED).

As equações diferenciais podem ser classificadas por tipo, ordem e linearidade. Quanto ao tipo podem ser ordinária ou parcial, na qual a equação que apresenta derivadas com relação a uma única variável independente é chamada de equação diferencial ordinária (EDO), no entanto, se a equação apresentar derivadas com relação a duas ou mais variáveis independentes é denominada de equação diferencial parcial (EDP). A classificação por ordem corresponde à derivada de maior ordem na equação. Quanto à linearidade, dada a EDO de n-ésima ordem por:

$$
a_{n}(x) \frac{d^{n} y}{d x^{n}}+a_{n-1}(x) \frac{d^{n-1} y}{d x^{n-1}}+a_{n-2}(x) \frac{d^{n-2} y}{d x^{n-2}}+\ldots+a_{1}(x) \frac{d y}{d x}+a_{0}(x)=g(x)
$$

A equação representada acima é considerada linear se $y$ e todas as suas derivadas são do primeiro grau e se os seus respectivos coeficientes $a_{0} \ldots a_{n}$ dependem de funções cuja variável é independente ou são constantes. Vale ressaltar também dois casos em que a Equação 01 pode ser apresentada, onde ela é dita homogênea se $g(x)$ $=0$, ou heterogênea se $g(x) \neq 0$. Nesse trabalho, as equações que serão estudadas devido às suas aplicações se resumem a equações diferenciais do tipo ordinária (EDO), lineares, homogêneas e de segunda ordem.

\subsubsection{Equação Linear de Primeira Ordem e de Segunda Ordem}

Uma equação linear de primeira ordem corresponde a uma equação cuja a ordem de sua maior derivada é igual a um, ou seja, a partir da Equação 01, ela será de primeira ordem quando $n=1$, descrevendo-se na forma:

$$
a_{1}(x) \frac{d y}{d x}+a_{0}(x) y=g(x)
$$

onde o coeficiente $a_{1}(x) \neq 0$.

Uma equação diferencial de segunda ordem é toda equação cuja a ordem de sua maior derivada é igual a dois, em outras palavras, tendo como base a Equação 01, ela será de segunda ordem se $n=2$ de forma que:

$$
a_{2}(x) \frac{d^{2} y}{d x^{2}}+a_{1}(x) \frac{d y}{d x}+a_{0}(x) y=g(x)
$$

onde o coeficiente $a_{2}(x) \neq 0$.

\subsubsection{Problema de Valor Inicial (PVI)}

Para se encontrar uma solução particular de uma EDO, ou seja, determinar uma função y(x) que satisfaça as determinadas condições de contorno que são impostas sobre ela e suas derivadas dada uma condição inicial $x_{0}$, é utilizado um problema denominado de problema de valor inicial (PVI). O problema de valor inicial para uma equação de $n$-ésima ordem está sujeita às seguintes condições iniciais:

$$
\mathrm{y}\left(\mathrm{x}_{0}\right)=\mathrm{y}_{0}, \quad \mathrm{y}^{\prime}\left(\mathrm{x}_{0}\right)=\mathrm{y}_{0}{ }^{\prime}, \ldots, \mathrm{y}^{(\mathrm{n}-1)}\left(\mathrm{x}_{0}\right)=\mathrm{y}_{0}^{(\mathrm{n}-1)}
$$

onde $\mathrm{x}_{0}, \mathrm{y}_{0}, y_{0}{ }^{\prime}, \ldots, \mathrm{y}^{(\mathrm{n}-1)}$ são conhecidos. No entanto, um PVI só terá solução única se a mesma seguir as condições do seguinte teorema:

Teorema 2.4: Sejam $\mathrm{a}_{2}(\mathrm{x}), \mathrm{a}_{1}(\mathrm{x}), \mathrm{a}_{0}(\mathrm{x})$ e $\mathrm{g}(\mathrm{x})$ contínuas no intervalo I e seja $\mathrm{a}_{2}(\mathrm{x}) \neq 0$ para todo $x$ nesse intervalo. Se $\mathrm{x}=x_{0}$ for um ponto qualquer desse intervalo, então existe uma única solução $\mathrm{y}(\mathrm{x})$ do problema de valor inicial nesse intervalo.

Logo, para um problema de valor inicial de uma EDO de segunda ordem, temos que as condições iniciais são: 


$$
\mathrm{y}\left(\mathrm{x}_{0}\right)=\mathrm{y}_{0}, \mathrm{y}^{\prime}\left(\mathrm{x}_{0}\right)=\mathrm{y}_{0}^{\prime}
$$

sendo $\mathrm{x}_{0}, \mathrm{y}_{0}$ e $\mathrm{y}_{0}{ }^{\prime}$ dados conhecidos.

\subsection{Vibração Livre com Amortecimento Viscoso}

A vibração livre [2] é compreendida como a vibração que um sistema exerce como resultado de uma perturbação inicial (condições iniciais não nulas), que pode ser um impulso retirando-o da posição de equilíbrio ou um impacto que imprime uma velocidade inicial não nula. O sistema massa-mola, objeto de estudo do trabalho, é basicamente composto por um corpo que está preso em uma mola e que oscila livremente em meio viscoso após uma perturbação inicial por meio da distensão da mola (massa desprezível). Na Figura 1 é representado o sistema descrito. No balanço de forças, temos que o peso da massa do corpo (para baixo) gera uma deformação na mola na qual a mesma reage aplicando uma força elástica (para cima), havendo um equilíbrio de forças e chegando à posição de equilíbrio do sistema. No entanto, ao tratarmos o sistema perante a utilização de um fluido como amortecimento, haverá a presença de mais um componente de força que participará do equilíbrio. A posição do corpo que está oscilando perante uma perturbação inicial em um meio viscoso em um certo instante de tempo pode ser obtida a partir da utilização de equações diferenciais.

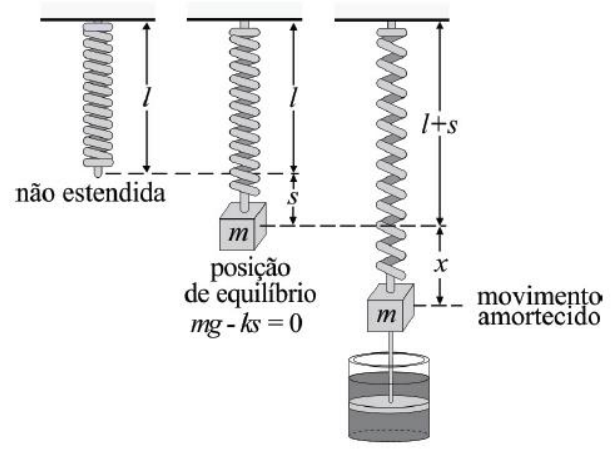

(a) (b)

(c)

FIGURA 1. Sistema massa-mola em diferentes estados. (a) mola não estendida, (b) posição de equilíbrio e (c) movimento amortecido. [6]

A equação que descreve a vibração livre com amortecimento viscoso é dado por:

$$
\mathrm{m} \frac{\mathrm{d}^{2} \mathrm{x}}{\mathrm{dt}^{2}}+\mathrm{c} \frac{\mathrm{dx}}{\mathrm{dt}}+\mathrm{kx}=0
$$

onde m é a massa do corpo, desprezando-se a massa da mola, c a constante de amortecimento, $\mathrm{k}$ a constante da mola e x a deformação da mola na perturbação. A sua equação auxiliar é expressa por:

cuja raízes são:

$$
\mathrm{ms}^{2}+\mathrm{cs}+\mathrm{k}=0
$$

$$
\mathrm{s}_{1,2}=\frac{-\mathrm{c}}{2 \mathrm{~m}} \pm \sqrt{\left(\frac{\mathrm{c}}{2 \mathrm{~m}}\right)^{2}-\frac{\mathrm{k}}{\mathrm{m}}},
$$

contudo, antes de encontrarmos as soluções, é interessante aprofundar um pouco o estudo e reescrever as raízes em função da constante de amortecimento crítico e o fator de amortecimento.

De acordo com [2], o amortecimento crítico $c_{c}$ é definido como o valor que a constante de amortecimento $c$ apresenta para que o valor do radicando da Equação 08 torne-se zero: 


$$
\begin{aligned}
& \left(\frac{\mathrm{c}_{\mathrm{c}}}{2 \mathrm{~m}}\right)^{2}-\frac{\mathrm{k}}{\mathrm{m}}=0 \\
& c_{c}=2 m \omega_{n},
\end{aligned}
$$

onde $\omega_{\mathrm{n}}=\sqrt{\frac{\mathrm{k}}{\mathrm{m}}}$, é a frequência natural de vibração do sistema.

$\mathrm{O}$ fator de amortecimento $\zeta$ é definido pela razão entre a constante de amortecimento $c$ e o amortecimento crítico $c_{c}$ da seguinte forma:

$$
\zeta=\frac{\mathrm{c}}{\mathrm{c}_{\mathrm{c}}}
$$

Podemos reescrever as raízes como:

$$
\mathrm{s}_{1,2}=\left(-\zeta \pm \sqrt{\zeta^{2}-1}\right) \omega_{\mathrm{n}}
$$

assumindo $0 \leq \zeta \leq \infty$, o radicando na expressão das raízes pode ser positivo, negativo ou zero, o que nos leva ao estudo de três casos possíveis para o comportamento do fenômeno: sistema superamortecido $(\zeta>1)$, que descreve um movimento aperiódico no qual para o tempo tendendo ao infinito $(t \rightarrow \infty)$, a posição do corpo retornará para zero; sistema criticamente amortecido $(\zeta=1)$, consiste no princípio básico de funcionamento de um amortecedor, uma vez que neste caso, a função tende a zero em um intervalo de tempo muito menor e o seu movimento é aperiódico; sistema subamortecido $(\zeta<1)$, onde o movimento desse sistema é oscilatório, no entanto, sua amplitude diminui exponencialmente com o tempo levando-o à posição zero.

\subsection{Polinômios de Taylor}

Antes de introduzir os métodos numéricos à serem trabalhados, é importante conceituar o Polinômio de Taylor, pois o mesmo tem aplicação importante na resolução numérica de equações diferenciais.

O teorema de Taylor [1] afirma que qualquer função suave pode ser aproximada por um polinômio, visto que os polinômios apresentam derivadas e integrais elementares. Em outras palavras, o teorema de Taylor consiste em representar funções em séries de potência em $a$ para uma análise numérica, onde:

$$
f(x)=f(a)+f(a)+\frac{f^{\prime}(a)}{1 !} \cdot(x-a)+\frac{f^{\prime \prime}(a)}{2 !} \cdot(x-a)^{2}+\ldots+\frac{f^{(n)}(a)}{n !} \cdot(x-a)^{n}
$$

Quando a Equação 12 é limitada em um termo finito, tem-se um Polinômio de Taylor, onde o resto da série é truncado e chamado de erro de truncamento local $\left(E T L_{I}\right)$. Logo, o polinômio de Taylor $(T(x))$ de ordem $i$ é dado por:

$$
T_{i}(x)=\sum_{n=0}^{i} \frac{f^{(n)}(a)}{n !} \cdot(x-a)^{n}
$$

Substituindo a Equação 13 na Equação 12, temos:

$$
\begin{gathered}
f(x)=T_{i}(x)+E L_{i} \\
E T L_{i}=O\left(h^{i+1}\right)
\end{gathered}
$$

com $\mathrm{i}=0,1, \ldots,(\mathrm{n}-1)$ e $h$ o tamanho do passo. 


\subsection{Método de Euler}

Segundo [5], o método de Euler, desenvolvido por Leonhard Euler, consiste em um método numérico capaz de encontrar de forma simplificada uma aproximação para a solução de uma equação diferencial de primeira ordem a partir de um problema de valor inicial dado em um intervalo $[a, b]$. Esse método também é chamado de método da reta tangente, pois sua abordagem é feita a partir da equação da reta tangente (Figura 2). Dada uma EDO do tipo da Equação 02 com seu respectivo PVI, o método retornará não a aproximação para a solução $\mathrm{y}(\mathrm{x})$, mas sim aproximações por pontos, chamados de pontos de malha, que devem estar igualmente distribuídos no devido intervalo. Segundo [1], a primeira derivada da função $\mathrm{y}=\mathrm{f}(\mathrm{x})$, em $x_{0}$, fornece uma estimativa direta da inclinação em $x_{0}$. Portanto, para determinar a projeção aproximada de um ponto seguinte $x_{1}$ em $y$, é feita uma estimativa através desta inclinação, que é definida pela função L(x) (Figura 2).

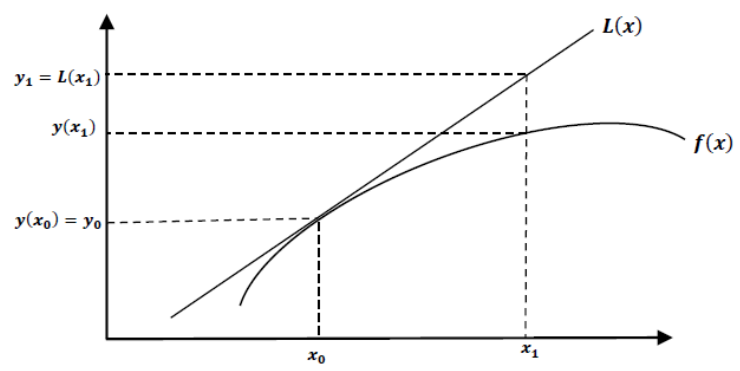

FIGURA 2. Gráfico das funções $\mathrm{L}(\mathrm{x})$ e $\mathrm{f}(\mathrm{x})$, onde $\mathrm{L}(\mathrm{x})$ é a reta tangente a $\mathrm{f}(\mathrm{x})$ no ponto $\left(\mathrm{x}_{0}, \mathrm{y}_{0}\right)$.[9]

Para se obter uma aproximação $\mathrm{y}_{\mathrm{i}}$ para um valor desconhecido $\mathrm{y}\left(\mathrm{x}_{\mathrm{i}}\right)$, é utilizada a equação da reta tangente $\mathrm{L}(\mathrm{x})$ na qual é representada, de forma genérica, pela Equação 16 considerando $\mathrm{h}=\frac{\mathrm{b} \text {-a }}{\mathrm{n}}$ chamado de tamanho de passo e $n$ um número inteiro positivo qualquer. Logo a funçao de iteração do método de Euler é dada por:

$$
\mathrm{y}_{\mathrm{i}+1}=\mathrm{y}_{\mathrm{i}}+\mathrm{hf}\left(\mathrm{x}_{\mathrm{i}}, \mathrm{y}_{\mathrm{i}}\right)
$$

\subsection{Método de Heun}

Segundo [1], ao considerar, no método de Euler, que a derivada no início do intervalo pode ser usada em todo o intervalo, poderá haver um considerável erro numérico. Portanto, o método de Heun, também chamado de Euler melhorado, apresenta uma estratégia para se encontrar a solução numérica com melhor precisão. Uma das estratégias, é determinar duas derivadas, uma no ponto inicial e outra no ponto final, onde a nova inclinação será dada pela média das duas derivadas. Logo, tomando a Equação 16, e adaptando para a estratégia descrita temos:

$$
\mathrm{y}_{\mathrm{i}+1}=\mathrm{y}_{\mathrm{i}}+\mathrm{h} \frac{\mathrm{f}\left(\mathrm{x}_{\mathrm{i}}, \mathrm{y}_{\mathrm{i}}\right)+\mathrm{f}\left(\mathrm{x}_{\mathrm{i}+1}, \mathrm{y}_{\mathrm{i}+1}\right)}{2}
$$

A Figura 3 ilustra graficamente o funcionamento do método de Heun.

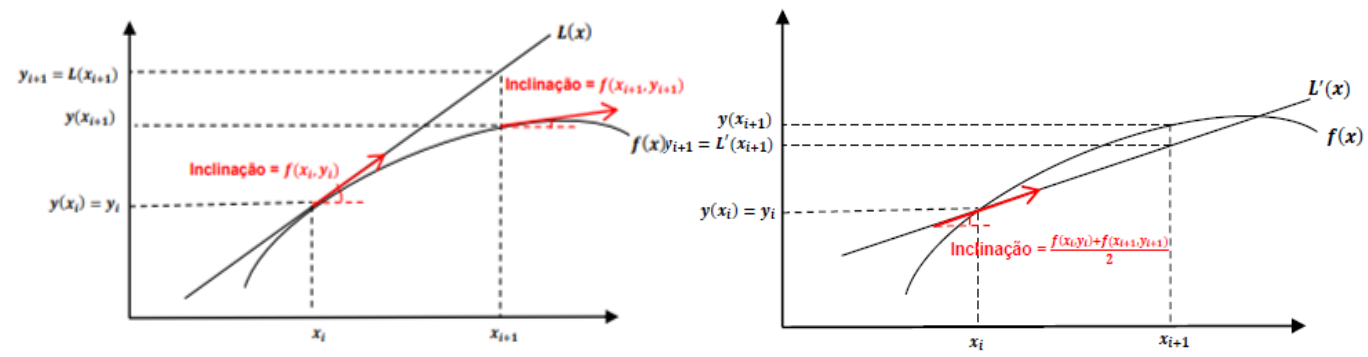

Figura 3. Demonstração gráfica do método de Heun.[9] 


\subsection{Método de Runge-Kutta}

Desenvolvido por Carl David Runge e M. Wilhelm Kutta, o método é um dos mais eficientes e populares para a solução de problemas de valor inicial. Segundo [5], "os métodos de Runge-Kutta (RK) alcançam a acurácia de uma abordagem por série de Taylor sem exigir cálculos de derivadas de ordem superior", justificando então sua acurácia. Os métodos têm como base os polinômios de Taylor e são classificados quanto à ordem, onde a mesma é definida pelo $n$ na função. O método Runge-Kutta de n-ésima ordem é dado por:

$$
\mathrm{y}_{\mathrm{i}+1}=\mathrm{y}_{\mathrm{i}}+\mathrm{h} \cdot\left(\sum_{\mathrm{i}=1}^{\mathrm{n}} \mathrm{a}_{\mathrm{i}} \mathrm{k}_{\mathrm{i}}\right)
$$

no qual $\mathrm{a}_{\mathrm{i}}$ e $\mathrm{k}_{\mathrm{i}}$ são respectivamente constantes e funções relacionadas ao problema em questão.

O método RK de $1^{\mathrm{a}}$ ordem representa o método de Euler, na qual a série de Taylor é expandida até $n=1$ e o resto da sequência é truncado, bem como o método RK de $2^{\mathrm{a}}$ ordem representa o método de Heun, na qual a série de Taylor é expandida até $n=2$ e o resto da sequência é truncado. Tendo em vista que quanto maior a ordem menor será o erro por truncamento [1], o método mais utilizado por possuir maior precisão bem como ter uma simples implementação, é o método de RK de quarta ordem, que é dado por:

$$
\mathrm{y}_{\mathrm{i}+1}=\mathrm{y}_{\mathrm{i}}+\frac{\mathrm{h}}{6}\left(\mathrm{k}_{1}+2 \mathrm{k}_{2}+2 \mathrm{k}_{3}+\mathrm{k}_{4}\right)
$$

com $\mathrm{i}=0,1, \ldots,(\mathrm{n}-1), \mathrm{e}:$

$$
\begin{gathered}
\mathrm{k}_{1}=\mathrm{f}\left(\mathrm{x}_{\mathrm{i}}, \mathrm{y}_{\mathrm{i}}\right) \\
\mathrm{k}_{2}=\mathrm{f}\left(\mathrm{x}_{\mathrm{i}}+\frac{\mathrm{h}}{2}, \mathrm{y}_{\mathrm{i}}+\frac{\mathrm{h}}{2} \mathrm{k}_{1}\right) \\
\mathrm{k}_{3}=\mathrm{f}\left(\mathrm{x}_{\mathrm{i}}+\frac{\mathrm{h}}{2}, \mathrm{y}_{\mathrm{i}}+\frac{\mathrm{h}}{2} \mathrm{k}_{2}\right) \\
\mathrm{k}_{4}=\mathrm{f}\left(\mathrm{x}_{\mathrm{i}}+\mathrm{h}, \mathrm{y}_{\mathrm{i}}+\mathrm{hk}_{3}\right)
\end{gathered}
$$

As equações mostradas acima correspondem a uma solução particular uma vez que existem outros métodos de Runge-Kutta de quarta ordem geradas a partir da variação dos coeficientes do método.

\section{Metodologia}

De modo a verificar a eficiência dos métodos de Euler, Heun e Runge-Kutta na resolução de problemas de valor inicial de equações diferenciais que descrevem problemas de sistema massa-mola com três casos diferentes de amortecimento, foi realizada a simulação numérica dos métodos numéricos no IDE SciLab versão 5.5.2, utilizando um intervalo de [0,2] na posição, e posteriormente os resultados foram comparados com a função solução, encontrada em [6] de forma analítica, de cada problema. Nos testes, foi realizada a análise da influência do passo $h$ na aproximação da solução e, como critérios de análise da eficiência dos métodos foram utilizados o erro absoluto (Equação 24) e o erro relativo (Equação 25) bem como a análise gráfica.

$$
\begin{aligned}
& E_{\text {absoluto }}=\left|y_{i+1}-y_{i}\right| \times 100 \% \\
& E_{\text {relativo }}=\frac{\left|y_{i+1}-y_{i}\right|}{y_{i+1}} \times 100 \%
\end{aligned}
$$




\subsection{Problemas Teóricos}

Os problemas que serão solucionados [6] abordam os três tipos (casos) de vibração amortecida que foram vistos nesse trabalho.

Problema 1: Sistema superamortecido. A equação diferencial e seu respectivo PVI são dados por:

$$
\begin{gathered}
1,2 x^{\prime \prime}+100 x^{\prime}+180 x=0 \\
\left\{\begin{array}{c}
x(0)=0,15 \mathrm{~m} \\
x^{\prime}(0)=0 \mathrm{~m} / \mathrm{s}
\end{array}\right.
\end{gathered}
$$

Problema 2: Sistema criticamente amortecido. A equação diferencial e seu respectivo PVI são dados por:

$$
\begin{gathered}
1,2 x^{\prime \prime}+29,4 x^{\prime}+180 x=0 \\
\left\{\begin{array}{l}
x(0)=0,15 \mathrm{~m} \\
x^{\prime}(0)=0 \mathrm{~m} / \mathrm{s}
\end{array}\right.
\end{gathered}
$$

Problema 3: Sistema subamortecido. A equação diferencial e seu respectivo PVI são dados por:

$$
\begin{gathered}
1,2 x^{\prime \prime}+3 x^{\prime}+180 x=0 \\
\left\{\begin{array}{l}
x(0)=0,15 \mathrm{~m} \\
x^{\prime}(0)=0 \mathrm{~m} / \mathrm{s}
\end{array}\right.
\end{gathered}
$$

Tendo em vista que os problemas acimas são representados por equações diferenciais de segunda ordem, e os respectivos métodos numéricos utilizados apenas se aplicam a equações de primeira ordem, é necessário então que haja a redução da mesma para sua posterior execução.

\subsection{Redução de ordem}

Para reduzir a ordem da EDO, foi utilizado uma técnica que, tendo inicialmente uma equação diferencial de segunda ordem homogênea e seus respectivo PVI, temos:

$$
\begin{gathered}
y^{\prime \prime}+a_{1} y^{\prime}+a_{0} y=0 \\
\left\{\begin{array}{l}
y\left(x_{0}\right)=y_{0} \\
y^{\prime}\left(x_{0}\right)=y_{1}
\end{array}\right.
\end{gathered}
$$

com $a_{1}$ e $a_{0}$ constantes quaisquer, podemos fazer a mudança de variável (redução de ordem):

$$
\mathrm{z}^{\prime}=\mathrm{y}^{\prime} \mathrm{e} \mathrm{z}^{\prime}=\mathrm{y}^{\prime \prime}
$$

e reescrever a equação e seu respectivo PVI como:

$$
\begin{gathered}
z^{\prime}+a_{1} z+a_{0} y=0 \\
\left\{\begin{array}{l}
y\left(x_{0}\right)=y_{0} \\
z\left(x_{0}\right)=y_{1}
\end{array}\right.
\end{gathered}
$$

logo, chegamos a duas equações diferenciais de primeira ordem com seus respectivos PVI [7]. Uma vez que a redução é realizada, as equações se tornam propícias à utilização dos métodos numéricos, no entanto, os algoritmos dos mesmos devem passar por uma manipulação de forma a resolverem os problemas retornando valores em forma de vetor duplo $\left(\begin{array}{l}y \\ z\end{array}\right)$. 


\section{RESULTADOS E DISCUSSÃO}

\subsection{Problema 1}

Reduzindo a ordem da equação segundo a seção 3.2, temos:

$$
\begin{gathered}
1,2 z^{\prime}+100 z+180 x=0 \\
\left\{\begin{array}{c}
x(0)=0,15 \mathrm{~m} \\
z(0)=0 \mathrm{~m} / \mathrm{s}
\end{array}\right.
\end{gathered}
$$

De acordo com [6], a solução analítica do respectivo problema é dada por:

$$
x(t)=0,1535 \mathrm{e}^{-1,8406 \mathrm{t}}-0,0034 \mathrm{e}^{-81,4931 t}
$$

Os resultados obtidos a partir da execução do método nos algoritmos estão representados na Tabela 1 e na Figura 4.
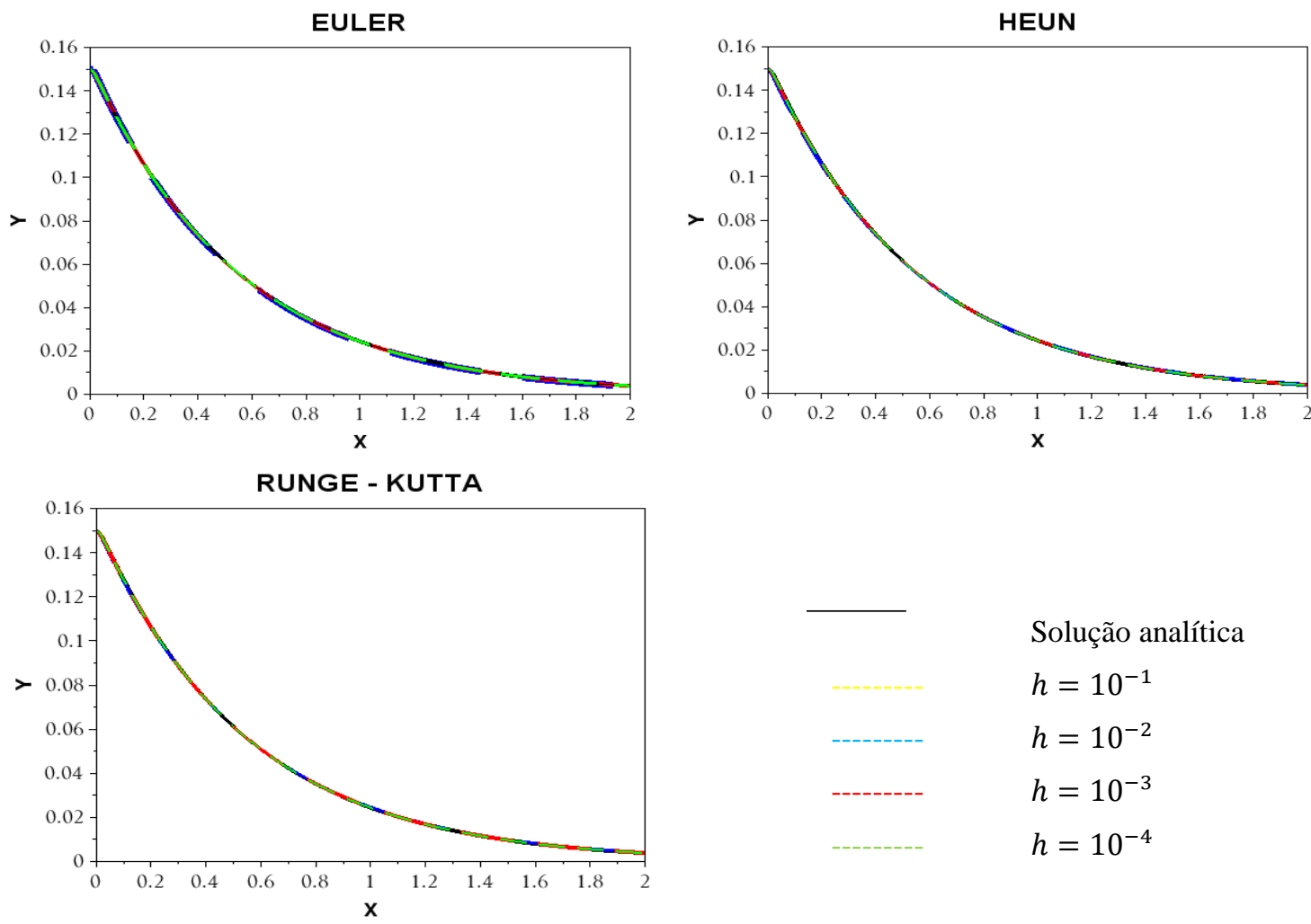

Solução analítica

$h=10^{-1}$

$h=10^{-2}$

$h=10^{-3}$

$h=10^{-4}$

FIGURA 4. Gráficos com as curvas estimadas em cada método de acordo com cada tamanho de passo. (Autoria própria) 
TABELA 1. Erro absoluto e Erro relativo médio dos resultados dos testes de cada método para cada valor de $h$.

\begin{tabular}{|c|c|c|c|}
\hline \multicolumn{4}{|c|}{ Erro absoluto médio } \\
\hline$h$ & Euler & Heun & Runge-Kutta \\
\hline 0,1 & ñ estima & ñ estima & ñ estima \\
\hline 0,01 & $3,5390 \times 10^{-4}$ & $3,5390 \times 10^{-4}$ & $2,9988 \times 10^{-4}$ \\
\hline 0,001 & $4,3396 \times 10^{-5}$ & $3,3915 \times 10^{-5}$ & $3,4628 \times 10^{-5}$ \\
\hline 0,0001 & $1,3490 \times 10^{-5}$ & $8,8650 \times 10^{-6}$ & $8,8542 \times 10^{-6}$ \\
\hline \multicolumn{4}{|c|}{ Erro relativo médio (\%) } \\
\hline$h$ & Euler & Heun & Runge-Kutta \\
\hline 0,1 & $\tilde{n}$ converge & $\tilde{n}$ converge & ñ converge \\
\hline 0,01 & 1,7267 & 0,8931 & 1,523 \\
\hline 0,001 & 0,1972 & 0,1603 & 0,1667 \\
\hline 0,0001 & 0,0452 & 0,0134 & 0,0134 \\
\hline
\end{tabular}

Segundo os gráficos e a tabela acima, os métodos numéricos foram eficientes na estimativa da função solução, uma vez que as curvas dos mesmos se aproximaram de forma satisfatória da curva da solução analítica. Vale salientar também, os pequenos valores dos erros absolutos médios e dos erros relativos médios, que reforça a ideia da eficiência dos métodos. Foi possível verificar que quanto menor o tamanho do passo h, melhor é a estimativa da solução em todos os métodos.

\subsection{Problema 2}

Reduzindo a ordem da equação segundo a seção 3.2, temos:

$$
\begin{gathered}
1,2 z^{\prime}+29,4 z+180 x=0 \\
\left\{\begin{array}{c}
x(0)=0,15 \mathrm{~m} \\
z(0)=0 \mathrm{~m} / \mathrm{s}
\end{array}\right.
\end{gathered}
$$

De acordo com [6], a solução analítica do respectivo problema é dada pela Equação 42. Os resultados obtidos a partir da execução dos métodos estão representadas na Tabela 2 e na Figura 5.

$$
x(t)=0,15 e^{-12,2474 t}-0,0034 \cdot t \cdot e^{-12,2474 t}
$$



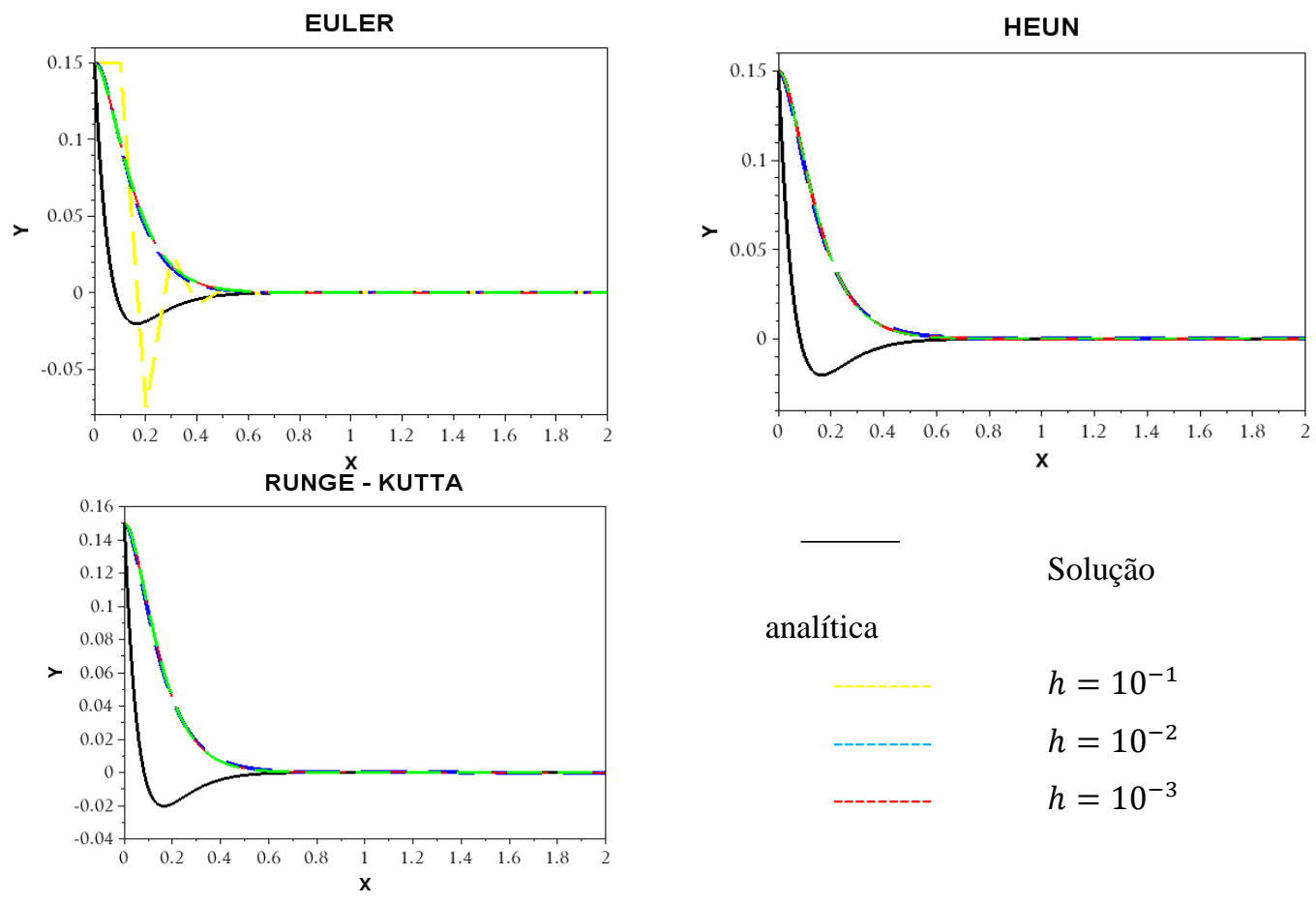

FIGURA 5: Gráficos com as curvas estimadas em cada método de acordo com cada tamanho de passo. (Autoria Própria)

TABELA 2. Erro absoluto e Erro relativo médio dos resultados dos testes de cada método para cada valor de h.

\begin{tabular}{cccc}
\hline \multicolumn{4}{c}{ Erro absoluto médio } \\
\hline$h$ & Euler & Heun & Runge-Kutta \\
\hline 0,1 & $1,2417 \times 10^{-2}$ & ñ estima & ñ estima \\
\hline 0,01 & $1,1801 \times 10^{-2}$ & $1,2180 \times 10^{-2}$ & $1,2217 \times 10^{-2}$ \\
\hline 0,001 & $1,2206 \times 10^{-2}$ & $1,2249 \times 10^{-2}$ & $1,2250 \times 10^{-2}$ \\
\hline 0,0001 & $1,2246 \times 10^{-2}$ & $1,2250 \times 10^{-2}$ & $1,2250 \times 10^{-2}$ \\
\hline \multicolumn{4}{c}{ Erro relativo médio $(\%)$} \\
\hline$h$ & Euler & Heun & Runge-Kutta \\
\hline 0,1 & 192,4739 & $\tilde{\mathrm{n}}$ estima & $\tilde{\mathrm{n}}$ estima \\
\hline 0,01 & 249,0492 & 1244,5104 & 1295,5445 \\
\hline 0,001 & 310,8966 & 362,0443 & 362,106 \\
\hline 0,0001 & 351,7262 & 356,4363 & 356,4367 \\
\hline
\end{tabular}

Podemos ver, segundo os gráficos e a tabela acima, que os métodos numéricos não foram eficientes na estimativa da função solução no momento em que houve o amortecimento, uma vez que as curvas dos mesmos não se aproximaram de forma satisfatória da curva da solução analítica. No entanto, o método conseguiu acompanhar a curva logo depois da perturbação, o que proporcionou uma melhora nos erros relativos médios. Foi possível verificar também, que no método de Euler quanto maior o tamanho do passo $h$ melhor a estimativa da solução, ao contrário do que ocorre no método de Heun e Runge-Kutta. 


\subsection{Problema 3}

Reduzindo a ordem da equação segundo a seção 3.2, temos:

$$
\begin{gathered}
1,2 z^{\prime}+3 z+180 x=0 \\
\left\{\begin{array}{c}
x(0)=0,15 \mathrm{~m} \\
z(0)=0 \mathrm{~m} / \mathrm{s}
\end{array}\right.
\end{gathered}
$$

De acordo com [6], a solução analítica do respectivo problema é dada por:

$$
x(t)=0,1508 e^{-1,2504 t} \cdot \operatorname{sen}(12,1834 t+1,4685)
$$

Os resultados obtidos a partir da execução do método nos algoritmos estão representados na Tabela 3 e na Figura 6.

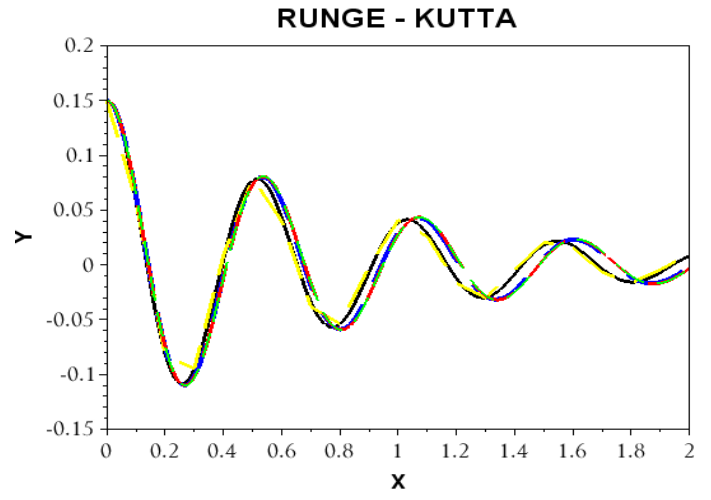

EULER

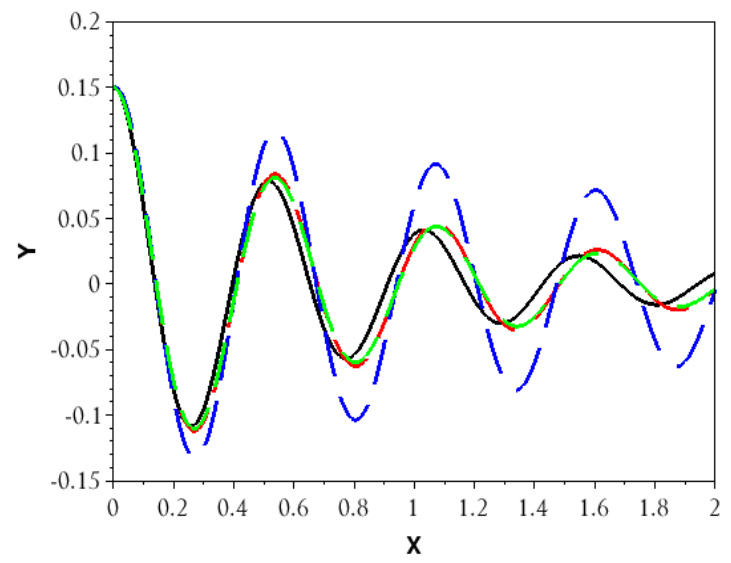

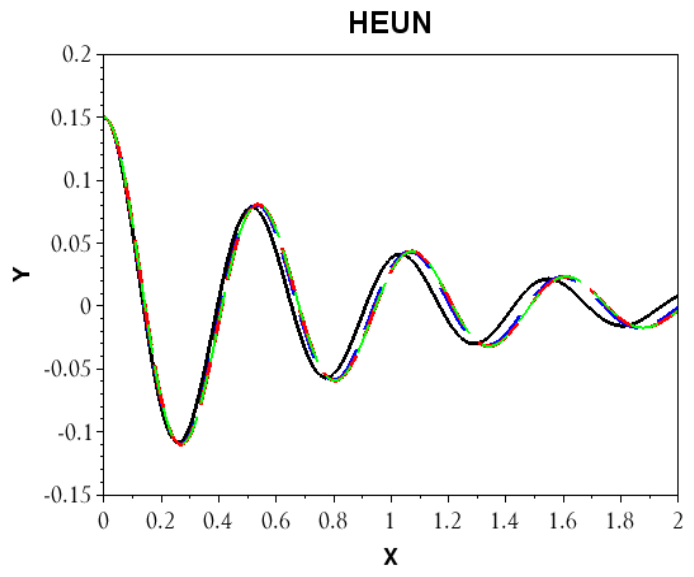

Solução analítica

$h=10^{-1}$

$h=10^{-2}$

$h=10^{-3}$

$h=10^{-4}$

FIGURA 6. Gráficos com as curvas estimadas em cada método de acordo com cada tamanho de passo. (Autoria Própria) 
TABELA 3. Erro absoluto e Erro relativo médio dos resultados dos testes de cada método para cada valor de h

\begin{tabular}{cccc}
\hline \multicolumn{4}{c}{ Erro absoluto médio } \\
\hline$h$ & Euler & Heun & Runge-Kutta \\
\hline 0,1 & $\tilde{\mathrm{n}}$ estima & 0,3459 & $2,2563 \times 10^{-3}$ \\
\hline 0,01 & $3,0266 \times 10^{-2}$ & $8,6940 \times 10^{-3}$ & $9,3054 \times 10^{-3}$ \\
\hline 0,001 & $1,1500 \times 10^{-2}$ & $1,0758 \times 10^{-2}$ & $1,0764 \times 10^{-2}$ \\
\hline 0,0001 & $1,0972 \times 10^{-2}$ & $1,0911 \times 10^{-2}$ & $1,0911 \times 10^{-2}$ \\
\hline \multicolumn{4}{c}{ Erro relativo médio $(\%)$} \\
\hline$h$ & Euler & Heun & Runge-Kutta \\
\hline 0,1 & $\tilde{\mathrm{n}}$ estima & 2936,7462 & 21,8877 \\
\hline 0,01 & 416,5053 & 138,6138 & 147,9508 \\
\hline 0,001 & 279,9656 & 259,1298 & 259,2719 \\
\hline 0,0001 & 503,7248 & 500,2132 & 500,216
\end{tabular}

De acordo com resultados, podemos notar que os métodos, no início da execução, conseguem acompanhar a curva da solução analítica. No entanto, ao passo que o processo é executado, os métodos passam a encontrar dificuldades na estimativa da curva tendo em vista o defasamento exposto. Essa dificuldade pode ser explicada pela curva da solução analítica se tratar de uma função senóide na qual seu caráter oscilatório dificulta a estimativa da curva pelos métodos [8]. De acordo com os resultados, não há um padrão na interferência do tamanho do passo $h$ nas estimativas da solução, salvo no método de Runge-Kutta que quanto maior o tamanho do passo melhor era a estimativa.

\section{CONCLUSÃO}

A partir do estudo e da análise da eficiência dos métodos numéricos iterativos na resolução de equações diferenciais que descrevem problemas de sistema massa-mola com três tipos de amortecimentos diferentes, pudemos concluir que os métodos se mostraram, no geral, eficientes na estimativa da solução no sistema superamortecido (Problema 1), destacando-se o método de Heun, e no sistema subamortecido (Problema 3), destacando-se o Método de Runge - Kutta. Já no sistema criticamente amortecido (Problema 2) os métodos não foram aceitáveis na aproximação da curva. Vale ressaltar que a utilização da manipulação de redução da EDO vista na seção 4.2, possivelmente contribuiu para o acúmulo de erro, dificultando a estimativa das curvas pelos métodos. É importante salientar também que o fato da redução do tamanho de passo não ter ocasionado o aumento da precisão e consequentemente diminuído o erro na maioria dos casos, além de o método de RungeKutta não se sair melhor que os outros como deveria ocorreu, provavelmente, pelo fato de que os métodos estão sendo aplicados no espaço tridimensional $(\mathrm{x}, \mathrm{y}, \mathrm{z})$, porém analisados em um espaço bidimensional por redução de ordem.

O respectivo trabalho é fruto de um projeto de pesquisa realizado nos últimos dois anos e com ele foi possível compreender melhor o comportamento dos métodos em diferentes situações, bem como pôde também explicitar limitações das aplicações dos mesmos e possíveis formas de suprir essas limitações. Com os devidos conhecimentos adquiridos no desenvolvimento desse trabalho bem como os resultados e conclusões obtidos dos testes, torna-se propício a continuação dos estudos nessa linha de pesquisa de modo a se trabalhar com outros métodos numéricos (diferenças finitas por exemplo) bem como o estudo e testes de outras formas de aproximação. 


\section{REFERÊNCIAS}

[1] CHAPRA, Steven C.. Métodos Numéricos Para Engenharia. 5. ed. Porto Alegre: Mc Graw Hill, 2008. 832 p.

[2] RAO, Singiresu S.. Vibrações Mecânicas. 4. ed. São Paulo: Pearson, 2008. 424p. Tradução de: Arlete Simille.

[3] ZILL, Dennis G.. Equações diferenciais com aplicações em modelagem. 10. ed. São Paulo: Cengage Learning, 2016. 437p. Tradução de: Márcio Koji Umezawa.

[4] BARROSO, Leônidas Conceição et al. Cálculo Numérico: (com Aplicações). 2. ed. São Paulo: Harbra, 1987. 384 p.

[5] BURDEN, R. L., FAIRES, D. Análise Numérica. 6.ed. São Paulo: Cengage Learning, 2010.

[6] FREITAS, Igor R. B. de; MENEZES, Matheus da Silva. ANÁLISE DIFERENCIAL DA VIBRAÇÃO LIVRE COM AMORTECIMENTO VISCOSO DE UM SISTEMA MASSA-MOLA PARA DIFERENTES FLUIDOS. 2018. 11 f. TCC (Graduação) - Curso de Ciência e Tecnologia, Universidade Federal Rural do SemiÁrido, Mosoró, 2018.

[7] VALLE, Marcos Eduardo. Métodos Numéricos para Sistemas de Equações Diferenciais e Equações de Ordem Superior: Campinas: Visual, 2015. 21 slides, color. Disponível em: <https://www.ime.unicamp.br/ valle/Teaching/2015/MS211/Aula14.pdf>. Acesso em: 26 ago. 2018.

[8] MAZURECHEN, Willington. Implementação computacional de métodos numéricos na resolução de E.D.O’S. 2016. 61 f. TCC (Graduação) - Curso de Licenciatura em Matemática, Universidade Estadual do Pará, União da Vitória, 2016.

[9] MEDONÇA, Pedro Thiago Vilela de. Comparativo entre os métodos numéricos de Euler, Heun e RungeKutta na resolução de problemas de valor inicial de primeira ordem. 2016. 56 f. TCC (Graduação) - Curso de Ciência e Tecnologia, Universidade Federal Rural do Semi-Árido, Angicos, 2016. 


\title{
Artigo \\ Aperfeiçoamento de algoritmos de sincronização de registros oscilográficos baseados em de tensão e corrente pré-falta
}

\author{
Jackson Nogueira Feitosa ${ }^{[1]}$, Melinda Cesianara Silva da Cruz ${ }^{[2]}$ \\ ${ }^{[1]}$ Universidade Federal Rural do Semi-árido; jacksonfeitosa16@gmail.com \\ ${ }^{[2]}$ Universidade Federal Rural do Semi-árido; melinda@ufersa.edu.br \\ Recebido: 10/07/2019; \\ Aceito: $12 / 07 / 2019$; \\ Publicado: 07/10/2019.
}

Resumo: Algoritmos de sincronização de registros oscilográficos são ferramentas importantes para a operação de sistemas de potência, sendo capazes de determinar o ângulo de correção necessário para alimentar, por exemplo, softwares de localização de faltas em linhas de transmissão e distribuição de energia. Existem na literatura vários métodos que fazem a sincronização de registros, sendo que os métodos mais vantajosos são aqueles que independem das características elétricas da linha como resistência, indutância e capacitância, as quais podem conter erros. Este trabalho propõe realizar uma análise e sugerir melhorias na implementação de duas metodologias de sincronização de registros oscilográficos existentes na literatura, ambas baseadas em fasores de tensão e corrente pré-falta, e independentes dos parâmetros elétricos da linha. Nos testes efetuados foram utilizados fasores de tensão e corrente obtidos a partir de simulações realizadas nos softwares ATPDraw e ANAREDE.

Palavras-chave: Sincronização de registros oscilográficos; localização de faltas; linhas de transmissão; parâmetros elétricos.

\section{INTRODUÇÃO}

São encontrados na literatura diversos estudos sobre a localização de faltas em linhas de transmissão

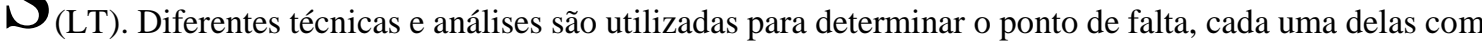
suas vantagens e desvantagens. Porém, essas técnicas, em sua maioria, necessitam de mecanismos capazes de sincronizar dados de tensão e corrente dos dois terminais da linha [1].

Relata-se em [2], que diversos estudiosos admitem empregar em suas análises, dados de tensão e corrente sincronizados, previamente, por PMUs (Phasor Measurement Units). PMUs são equipamentos que medem grandezas fasoriais utilizando o sistema de GPS (Global Positioning System) necessitando de um grande sistema de comunicação, o qual possui custos elevados. Inclusive, perdas no sinal de GPS podem acarretar em uma má sincronização de registros dos dados. Dessa forma, a sincronização de dados realizada via software ainda é um instrumento importante para auxiliar na extração correta de fasores necessários para determinar o local de uma falta em uma linha de transmissão.

Grande parte dos algoritmos que realizam a sincronização de registros de falta levam em consideração as componentes transitórias da linha de transmissão, destacando-se os fundamentados pelo uso da Transformada Walvelet [3] e algoritmos que comparam, em um sinal amostrado, valores instantâneos de dois ciclos consecutivos [4]. Encontram-se na literatura ainda técnicas que utilizam fasores de tensão e corrente pré-falta, dependentes dos parâmetros elétricos da linha [5], e não dependentes desses parâmetros [1-2]. Vale salientar que os parâmetros elétricos das linhas de transmissão podem conter erros ou podem estar indisponíveis 
computacionalmente, sendo relevante o uso de ferramentas que independam desses dados, uma vez que isso significa a exclusão de uma possível fonte de erros.

Este artigo apresenta uma modificação nas metodologias apresentadas em [1] e [2] para a determinação do ângulo de sincronização de registros, visto que o primeiro algoritmo apresentou falhas em algumas situações de operação do sistema e o segundo apresenta um número excedente de condições e equações para a determinação do ângulo correto de sincronização. Portanto, o novo algoritmo que vêm a ser proposto neste trabalho, resultante do aperfeiçoamento dos métodos descritos em [1] e [2], continua identificando os ângulos de sincronização em qualquer quadrante, e apresentando o benefício de operar corretamente em qualquer condição de operação do sistema elétrico com um menor número de condições a serem atendidas.

O procedimento sugerido bem como as duas metodologias analisadas, independe de parâmetros elétricos e características transitórias da linha de transmissão, pois o mesmo necessita apenas dos fasores de tensão e corrente pré-falta. Inclusive, a metodologia por depender destes dados pode ser aplicada em casos de difícil sincronização, onde existam parâmetros de linha desconhecidos ou inexatos, e registros com características transitórias atípicas [1]. Neste estudo foi realizada uma análise de desempenho da metodologia proposta com base em dados simulados no software ATPDraw (Alternative Transient Program) e no software ANAREDE (Análise de Redes Elétricas).

\section{ALGORITMOS DE SINCRONIZAÇÃO DE REGISTROS DE FALTA ANALISADOS}

A seguir será apresentado o equacionamento que constitui o algoritmo de sincronização de registros oscilográficos descrito em [1]. Para isso, considerar a Figura 1, que mostra o circuito equivalente de sequência positiva de uma linha de transmissão no instante pré-falta.

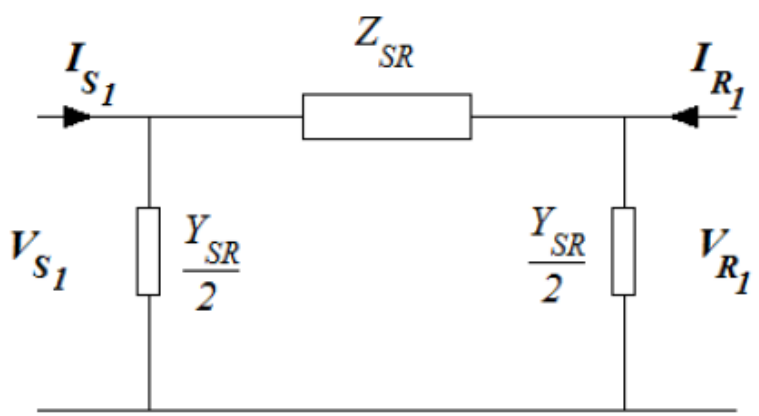

FIGURA 1. Circuito equivalente de sequência positiva de uma LT no instante pré-falta. [1]

Na Figura 1, os termos $Z_{S R}$ e $Y_{S R}$ representam, respectivamente, a impedância série e admitância shunt de sequência positiva da LT. Os termos $V_{S_{1}}, I_{S_{1}}, V_{R_{1}}, I_{R_{1}}$, representam, nesta ordem, os fasores de tensão e corrente pré-falta nos terminais $\mathrm{S}$ e $\mathrm{R}$, como também o índice 1 diz respeito à sequência positiva da linha de transmissão.

Da análise da Figura 1, obtém-se a Equação (1).

$$
\mathrm{I}_{\mathrm{S}_{1}}+\mathrm{I}_{\mathrm{R}_{1}} \mathrm{e}^{\mathrm{j} \delta}=\frac{\mathrm{Y}_{\mathrm{SR}}}{2}\left(\mathrm{~V}_{\mathrm{S}_{1}}+\mathrm{V}_{\mathrm{R}_{1}} \mathrm{e}^{\mathrm{j} \delta}\right)
$$

Sendo $\delta$ o ângulo sincronização entre os dois terminais descritos.

A Equação 1 é complexa e pode ser desmembrada em duas equações reais, conforme mostrado nas Equações 2 e 3 .

$$
\begin{gathered}
\mathrm{I}_{\mathrm{S}_{1}} \cos \left(\theta_{\mathrm{I}_{1}}\right)+\mathrm{I}_{\mathrm{R}_{1}} \cos \left(\theta_{\mathrm{I}_{\mathrm{R}_{1}}}\right)=\frac{-\mathrm{b}_{\text {shunt }}}{2}\left(\mathrm{~V}_{\mathrm{S}_{1}} \operatorname{sen}\left(\theta_{\mathrm{V}_{\mathrm{S}_{1}}}\right)+\mathrm{V}_{\mathrm{R}_{1}} \operatorname{sen}\left(\theta_{\mathrm{V}_{\mathrm{R}_{1}}}+\delta\right)\right) \\
\mathrm{I}_{\mathrm{S}_{1}} \operatorname{sen}\left(\theta_{\mathrm{I}_{\mathrm{S}_{1}}}\right)+\mathrm{I}_{\mathrm{R}_{1}} \operatorname{sen}\left(\theta_{\mathrm{I}_{\mathrm{R}_{1}}}\right)=\frac{-\mathrm{b}_{\text {shunt }}}{2}\left(\mathrm{~V}_{\mathrm{S}_{1}} \cos \left(\theta_{\mathrm{V}_{\mathrm{S}_{1}}}\right)+\mathrm{V}_{\mathrm{R}_{1}} \cos \left(\theta_{\mathrm{V}_{\mathrm{R}_{1}}}+\delta\right)\right)
\end{gathered}
$$

Adotando:

$$
\mathrm{a}=\mathrm{I}_{\mathrm{S}_{1}} \cos \left(\theta_{\mathrm{I}_{\mathrm{S}_{1}}}\right)
$$




$$
\begin{aligned}
\mathrm{b}=\mathrm{I}_{\mathrm{S}_{1}} \operatorname{sen}\left(\theta_{\mathrm{I}_{\mathrm{S}_{1}}}\right) \\
\mathrm{c}=\mathrm{V}_{\mathrm{S}_{1}} \operatorname{sen}\left(\theta_{\mathrm{V}_{\mathrm{S}_{1}}}\right) \\
\mathrm{d}=\mathrm{V}_{\mathrm{S}_{1}} \cos \left(\theta_{\mathrm{V}_{\mathrm{S}_{1}}}\right)
\end{aligned}
$$

As Equações 2 e 3 podem ser reescritas conforme as Equações 8 e 9.

$$
\begin{aligned}
& \mathrm{a}+\mathrm{I}_{\mathrm{R}_{1}} \cos \left(\theta_{\mathrm{I}_{1}}+\delta\right)=\frac{-\mathrm{b}_{\text {shunt }}}{2}\left(\mathrm{c}+\mathrm{V}_{\mathrm{R}_{1}} \operatorname{sen}\left(\theta_{\mathrm{V}_{\mathrm{R}_{1}}}+\delta\right)\right) \\
& \mathrm{b}+\mathrm{I}_{\mathrm{R}_{1}} \cos \left(\theta_{\mathrm{I}_{1}}+\delta\right)=\frac{-\mathrm{b}_{\text {shunt }}}{2}\left(\mathrm{~d}+\mathrm{V}_{\mathrm{R}_{1}} \cos \left(\theta_{\mathrm{V}_{\mathrm{R}_{1}}}+\delta\right)\right)
\end{aligned}
$$

Agregando as Equações 8 e 9, obtém-se a Equação 10, que por sua vez gera a Equação 11 que depende somente de uma incógnita $\delta$.

$$
\begin{gathered}
\frac{\mathrm{a}+\mathrm{I}_{\mathrm{R}_{1}} \cos \left(\theta_{\mathrm{I}_{1}}+\delta\right)=\frac{-\mathrm{b}_{\text {shunt }}}{2}\left(\mathrm{c}+\mathrm{V}_{\mathrm{R}_{1}} \operatorname{sen}\left(\theta_{\mathrm{V}_{\mathrm{R}_{1}}}+\delta\right)\right)}{\mathrm{b}+\mathrm{I}_{\mathrm{R}_{1}} \cos \left(\theta_{\mathrm{I}_{1}}+\delta\right)=\frac{-\mathrm{b}_{\text {shunt }}}{2}\left(\mathrm{~d}+\mathrm{V}_{\mathrm{R}_{1}} \cos \left(\theta_{\mathrm{V}_{\mathrm{R}_{1}}}+\delta\right)\right)} \\
\mathrm{A}=\left(\mathrm{x}_{1}+\mathrm{x}_{2}+\mathrm{x}_{3}+\mathrm{x}_{4}\right) \cos (\delta)-\left(-\mathrm{y}_{1}+\mathrm{y}_{2}-\mathrm{y}_{3}+\mathrm{y}_{4}\right) \operatorname{sen}(\delta)
\end{gathered}
$$

Na Equação 11:

$$
\begin{gathered}
\mathrm{A}=\mathrm{ad}+\mathrm{bc}+\mathrm{V}_{\mathrm{R}_{1}} \mathrm{I}_{\mathrm{R}_{1}} \cos \left(\theta_{\mathrm{V}_{\mathrm{R}_{1}}}-\theta_{\mathrm{I}_{\mathrm{R}_{1}}}\right) \\
\mathrm{x}_{1}=-\mathrm{aV} \mathrm{V}_{\mathrm{R}_{1}} \cos \left(\theta_{\mathrm{V}_{\mathrm{R}_{1}}}\right) ; \mathrm{y}_{1}=\mathrm{aV}_{\mathrm{R}_{1}} \operatorname{sen}\left(\theta_{\mathrm{V}_{\mathrm{R}_{1}}}\right) \\
\mathrm{x}_{2}=-\mathrm{bV}_{\mathrm{R}_{1}} \operatorname{sen}\left(\theta_{\mathrm{V}_{\mathrm{R}_{1}}}\right) ; \mathrm{y}_{2}=\mathrm{bV}_{\mathrm{R}_{1}} \cos \left(\theta_{\mathrm{V}_{\mathrm{R}_{1}}}\right) \\
\mathrm{x}_{3}=-\mathrm{dI}_{\mathrm{R}_{1}} \cos \left(\theta_{\mathrm{I}_{1}}\right) ; \mathrm{y}_{3}=\mathrm{dI}_{\mathrm{R}_{1}} \operatorname{sen}\left(\theta_{\mathrm{I}_{\mathrm{R}_{1}}}\right) \\
\mathrm{x}_{4}=-\mathrm{cI}_{\mathrm{R}_{1}} \operatorname{sen}\left(\theta_{\mathrm{I}_{1}}\right) ; \mathrm{y}_{3}=\mathrm{cI}_{\mathrm{R}_{1}} \cos \left(\theta_{\mathrm{I}_{\mathrm{R}_{1}}}\right)
\end{gathered}
$$

Fazendo $\mathrm{x}=\mathrm{x}_{1}+\mathrm{x}_{2}+\mathrm{x}_{3}+\mathrm{x}_{4}$ e $\mathrm{y}=-\mathrm{y}_{1}+\mathrm{y}_{2}-\mathrm{y}_{3}+\mathrm{y}_{4}$ obtém-se a Equação 17.

$$
\mathrm{A}=\mathrm{x} \cos (\delta)-\mathrm{y} \operatorname{sen}(\delta)
$$

A partir da Equação 17 é possível representar a relação ente $A, x$ e $y$ por meio do triângulo retângulo, mostrado na Figura 2.

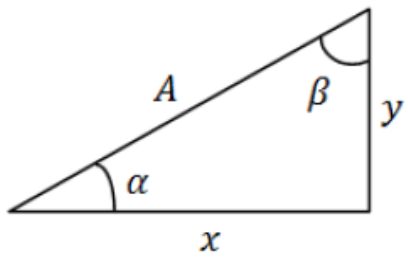

FIGURA 2. Relação geométrica ente A, y e x. [1]

A partir da análise conjunta da Equação 17 e da Figura 2, tem-se que: 


$$
\frac{\mathrm{A}}{\sqrt{\left(\mathrm{x}^{2}+\mathrm{y}^{2}\right)}}=\operatorname{sen}(\beta) \cos (\delta)-\operatorname{sen}(\delta) \cos (\beta)
$$

Dessa forma,

$$
\frac{\mathrm{A}}{\sqrt{\left(\mathrm{x}^{2}+\mathrm{y}^{2}\right)}}=\operatorname{sen}(\beta-\delta)
$$

A partir da Equação 19, pode-se determinar o ângulo de sincronização $\delta$, conforme a Equação 20.

$$
\delta=\operatorname{sen}^{-1}\left(\frac{x}{\sqrt{\left(x^{2}+y^{2)}\right.}}\right)-\operatorname{sen}^{-1}\left(\frac{A}{\sqrt{\left(x^{2}+y^{2}\right)}}\right)
$$

Considerando,

$$
\begin{aligned}
& \beta=\operatorname{sen}^{-1}\left(\frac{x}{\sqrt{\left(x^{2}+y^{2}\right)}}\right) \\
& \Phi=\operatorname{sen}^{-1}\left(\frac{A}{\sqrt{\left(x^{2}+y^{2}\right)}}\right)
\end{aligned}
$$

Assim,

$$
\delta=\beta-\Phi
$$

Em [1] para determinar o valor do ângulo $\delta$ no quadrante correto foi realizada uma análise sobre o sinal de $\mathrm{y}$, o que resultou no algoritmo a seguir.

$$
\begin{aligned}
& \text { Algoritmo: } \\
& \text { se }(y<0) \\
& \quad \delta=180^{\circ}-\beta-\Phi \\
& \text { senão } \\
& \quad \delta=\beta-\Phi \\
& \mathrm{fim}
\end{aligned}
$$

Para alguns casos de operação do sistema elétrico o algoritmo proposto em [1] expressou um ângulo de sincronização incorreto. Assim, [2] propôs uma nova metodologia, onde para se determinar o ângulo correto de sincronização é necessário considerar o módulo dos fasores de corrente em ambos os terminais, como mostrado a seguir.

$$
\begin{aligned}
& \text { Algoritmo: } \\
& \text { se }(x>0 \text { e } y>0) \\
& \text { se } I_{R_{1}}<I_{S_{1}} \\
& \delta=\beta_{1}-\Phi \\
& \text { fim } \\
& \text { se } I_{R_{1}}>I_{S_{1}} \\
& \delta=\beta_{1}-180^{\circ}+\Phi
\end{aligned}
$$




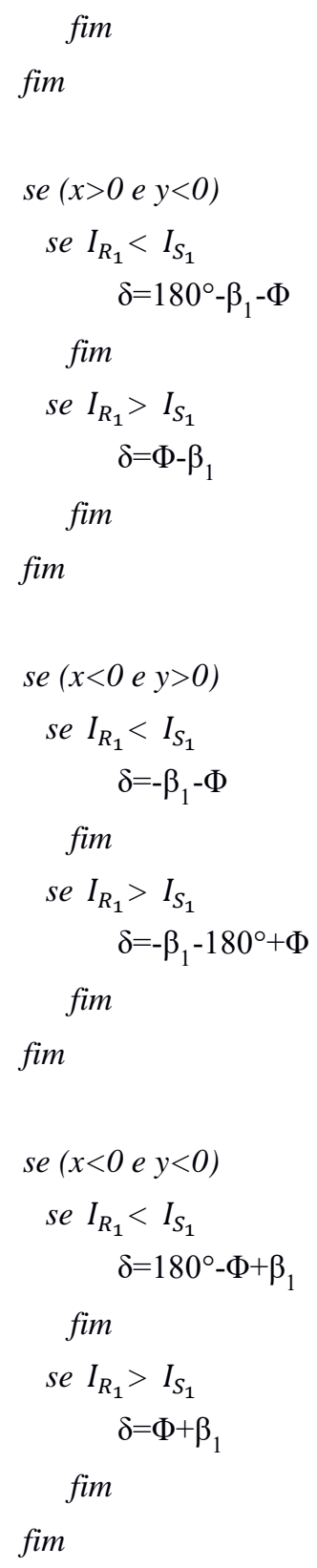

Vale salientar que $\beta_{1}$ no algoritmo acima é definido conforme a Equação 24.

$$
\beta_{1}=\operatorname{sen}^{-1}\left(\frac{|x|}{\sqrt{\left(x^{2}+y^{2}\right)}}\right)
$$

O algoritmo apresentado em [2] mostrou-se eficiente casos típicos de operação do sistema elétrico, determinando o ângulo de sincronização correto. Porém, ele possui um número elevado de equações e condições impostas para o sinal de $x$ que não existiam na metodologia descrita em [1].

Testes realizados no algoritmo proposto em [1] levaram ao novo algoritmo de sincronização proposto neste trabalho, que será apresentado na próxima seção. $\mathrm{O}$ algoritmo proposto é capaz de determinar o valor correto do ângulo de sincronização, para qualquer condição de operação do sistema, sem necessitar do conhecimento do sinal da variável $x$. Basicamente, conseguiu-se eliminar as limitações do algoritmo descrito em [1] apenas admitindo as condições do módulo de corrente descritas em [2].

Ressalta-se que em todas as metodologias de sincronização citadas neste trabalho, utiliza-se a Transformada Discreta de Fourier para a obtenção dos fasores de tensão e corrente pré-falta. 


\section{DEDUÇÃO DO ALGORITMO PROPOSTO}

Uma análise no algoritmo proposto em [1] foi efetuada para a dedução das equações e condições a serem utilizadas neste trabalho. Realizadas as simulações no ATPDraw e no ANAREDE, para determinadas situações de operação do sistema elétrico, foi analisado os valores absolutos das correntes $I_{R_{1}}$ e $I_{S_{1}}$, como também os sinais dos parâmetros x e y, e os ângulos $\beta$ e $\Phi$. Para isso foram escolhidos ângulos de sincronização de $120^{\circ} \mathrm{a}$ $-120^{\circ}$. As Tabelas 1,2 e 3 apresentam estes parâmetros para simulação de uma LT de $230 \mathrm{kV}$ considerando as situações de $0,95 \mathrm{pu}, 1 \mathrm{pu}$ e 1,05 pu no terminal $\mathrm{V}_{\mathrm{R}}$. As Tabelas 4 e 5 expressam os mesmos parâmetros para umaa $\mathrm{LT}$ real de $230 \mathrm{kV}$ e $500 \mathrm{kV}$, simulada no ANAREDE.

TABELA 1. Parâmetros e valores analisados para $\mathrm{V}_{\mathrm{R}}$ igual a 0,95 pu. (Autoria própria)

\begin{tabular}{|c|c|c|c|c|c|c|c|}
\hline \multicolumn{8}{|c|}{ Tensão 0,95 pu } \\
\hline \multirow{2}{*}{$\begin{array}{r}\hat{A} \text { ngulo de } \\
\text { sincronização } \\
\text { real (graus) }\end{array}$} & \multicolumn{6}{|c|}{ Parâmetros } & \multirow{2}{*}{$\begin{array}{c}\hat{A} \text { Angulo de } \\
\text { sincronização } \\
\text { calculado } \\
\text { (graus) }\end{array}$} \\
\hline & $\begin{array}{c}\beta \\
\text { (graus) }\end{array}$ & $\begin{array}{c}\Phi \\
\text { (graus) }\end{array}$ & $x$ & $y$ & $I_{R_{1}}(A)$ & $I_{S_{1}}(A)$ & \\
\hline 120 & $\begin{array}{c}- \\
66,2545 \\
\end{array}$ & 6,5293 & - & + & 296,5176 & 285,0606 & $-72,79$ \\
\hline 90 & $\begin{array}{c}- \\
83,7455\end{array}$ & 6,5293 & - & - & 296,5176 & 285,0606 & 257,21 \\
\hline 60 & $\begin{array}{c}- \\
53,7455 \\
\end{array}$ & 6,5293 & - & - & 296,5176 & 285,0606 & 227,21 \\
\hline 30 & $\begin{array}{c}- \\
23,7475 \\
\end{array}$ & 6,5293 & - & - & 296,5176 & 285,0606 & 197,21 \\
\hline 0 & 6,2545 & 6,5293 & + & - & 296,5176 & 285,0606 & 167,21 \\
\hline-30 & 36,2545 & 6,5293 & + & - & 296,5176 & 285,0606 & 137,21 \\
\hline-60 & 66,2545 & 6,5293 & + & - & 296,5176 & 285,0606 & 107,21 \\
\hline-90 & 83,7455 & 6,5293 & + & + & 296,5176 & 285,0606 & 77,21 \\
\hline-120 & 53,7455 & 6,5293 & + & + & 296,5176 & 285,0606 & 47,21 \\
\hline
\end{tabular}

TABELA 2. Parâmetros e valores analisados para $\mathrm{V}_{\mathrm{R}}$ igual a 1 pu. (Autoria própria)

\begin{tabular}{ccccccccc}
\hline \multicolumn{7}{c}{ Tensão l pu } \\
\hline $\begin{array}{c}\text { Anngulo de } \\
\text { sincronização } \\
\text { real (graus) }\end{array}$ & \begin{tabular}{c}
$\beta$ \\
\cline { 2 - 8 }
\end{tabular} & $\begin{array}{c}\text { Praumetros } \\
\text { (graus) }\end{array}$ & $x$ & $y$ & $I_{R_{1}}(A)$ & $I_{S_{1}}(A)$ & $\begin{array}{c}\text { Angulo de } \\
\text { sincronização } \\
\text { calculado } \\
\text { (graus) }\end{array}$ \\
\hline 120 & 56,0214 & 4,1682 & + & - & 277,1477 & 295,8820 & 119,81 \\
\hline 90 & 86,0214 & 4,1682 & + & - & 277,1477 & 295,8820 & 89,81 \\
\hline 60 & 63,9786 & 4,1682 & + & + & 277,1477 & 295,8820 & 59,81 \\
\hline 30 & 33,9786 & 4,1682 & + & + & 277,1477 & 295,8820 & 29,81 \\
\hline 0 & 3,9786 & 4,1682 & + & + & 277,1477 & 295,8820 & $-0,19$ \\
\hline-30 & $-26,0414$ & 4,1682 & - & + & 277,1477 & 295,8820 & $-30,19$ \\
\hline-60 & $-56,0214$ & 4,1682 & - & + & 277,1477 & 295,8820 & $-60,19$ \\
\hline-90 & $-86,214$ & 4,1682 & - & + & 277,1477 & 295,8820 & $-90,19$ \\
\hline-120 & $-63,9786$ & 4,1682 & - & - & 277,1477 & 295,8820 & 239,81 \\
\hline
\end{tabular}


TABELA 3. Parâmetros e valores analisados para $\mathrm{V}_{\mathrm{R}}$ igual a 1,05 pu. (Autoria própria)

\begin{tabular}{|c|c|c|c|c|c|c|c|}
\hline \multicolumn{8}{|c|}{ Tensão 1,05 pu } \\
\hline \multirow{2}{*}{$\begin{array}{l}\hat{A} n g u l o d e \\
\text { sincronização } \\
\text { real (graus) }\end{array}$} & \multicolumn{6}{|c|}{ Parâmetros } & \multirow{2}{*}{$\begin{array}{l}\hat{A} \text { Angulo de } \\
\text { sincronização } \\
\text { calculado } \\
\text { (graus) }\end{array}$} \\
\hline & $\beta$ (graus) & $\Phi$ (graus) & $x$ & $y$ & $I_{R_{1}}(A)$ & $I_{S_{1}}(A)$ & \\
\hline 120 & 58,3499 & 1,7228 & + & - & 279,8393 & 327,5798 & 119,95 \\
\hline 90 & 88,3499 & 1,7228 & + & - & 279,8393 & 327,5798 & 89,95 \\
\hline 60 & 61,6501 & 1,7228 & + & + & 279,8393 & 327,5798 & 59,95 \\
\hline 30 & 31,6501 & 1,7228 & + & + & 279,8393 & 327,5798 & 29,95 \\
\hline 0 & 1,6501 & 1,7228 & + & + & 279,8393 & 327,5798 & 0,045 \\
\hline-30 & $-28,3499$ & 1,7228 & - & + & 279,8393 & 327,5798 & $-30,05$ \\
\hline-60 & $-58,3499$ & 1,7228 & - & + & 279,8393 & 327,5798 & $-60,05$ \\
\hline-90 & $-88,3499$ & 1,7228 & - & + & 279,8393 & 327,5798 & $-90,05$ \\
\hline-120 & $-61,6501$ & 1,7228 & - & - & 279,8393 & 327,5798 & 239,95 \\
\hline
\end{tabular}

Assim percebeu-se que os casos onde o código proposto em [1] funciona quando os valores de $I_{S_{1}}$ foram maiores que os de $I_{R_{1}}$, porém isso não ocorre em casos contrários. A partir da análise dos dados, é possível concluir que isso ocorreu devido o sinal do ângulo $\beta$, para cada ângulo de sincronização, se inverter quando o valor de $I_{S_{1}}$ é menor que os de $I_{R_{1}}$. Dessa forma, é necessário analisar, além do sinal de y, também a grandeza dos valores absolutos de corrente pré-falta em ambos os terminais, como relatado em [2].

Diante disso, para a determinação do novo código foram preservadas as equações utilizadas pela metodologia proposta em [1], com o acréscimo de duas equações contendo as condições dos valores absolutos de corrente pré-falta em ambos os terminais.

Realizou-se esta mesma análise para as LTs de 230 e $500 \mathrm{kV}$, simuladas no software ANAREDE, onde notou-se que o padrão visto nos casos anteriores, se repete. Observa-se isso nas Tabelas 4 e 5.

TABELA 4. Parâmetros e valores analisados para LT real de 230 kV. (Autoria própria)

\begin{tabular}{|c|c|c|c|c|c|c|c|}
\hline \multicolumn{8}{|c|}{ Tensão $230 \mathrm{kV}$} \\
\hline \multirow{2}{*}{$\begin{array}{l}\text { Ângulo de } \\
\text { sincronização } \\
\text { real (graus) }\end{array}$} & \multicolumn{6}{|c|}{ Parâmetros } & \multirow{2}{*}{$\begin{array}{c}\text { Ângulo de } \\
\text { sincronização } \\
\text { calculado } \\
\text { (graus) }\end{array}$} \\
\hline & $\begin{array}{c}\beta \\
\text { (graus) }\end{array}$ & $\begin{array}{c}\Phi \\
\text { (graus) }\end{array}$ & $x$ & $y$ & $\begin{array}{c}I_{R_{1}} \\
(p u)\end{array}$ & $\begin{array}{c}I_{S_{1}} \\
(p u)\end{array}$ & \\
\hline 120 & 59,9198 & $-0,0494$ & + & - & 0,2500 & 0,2700 & 120,04 \\
\hline 90 & 89,9198 & $-0,0494$ & + & - & 0,2500 & 0,2700 & 90,04 \\
\hline 60 & 60,0802 & $-0,0494$ & + & + & 0,2500 & 0,2700 & 60,04 \\
\hline 30 & 30,0802 & $-0,0494$ & + & + & 0,2500 & 0,2700 & 30,04 \\
\hline 0 & 0,0802 & $-0,0494$ & + & + & 0,2500 & 0,2700 & 0,04 \\
\hline-30 & $-29,9198$ & $-0,0494$ & - & + & 0,2500 & 0,2700 & $-29,96$ \\
\hline-60 & $-59,9198$ & $-0,0494$ & - & + & 0,2500 & 0,2700 & $-59,96$ \\
\hline-90 & $-89,9198$ & $-0,0494$ & - & + & 0,2500 & 0,2700 & $-89,96$ \\
\hline-120 & $-60,0802$ & $-0,0494$ & - & - & 0,2500 & 0,2700 & 240,04 \\
\hline
\end{tabular}


TABELA 5. Parâmetros e valores analisados para LT real de 500 kV. (Autoria própria)

\begin{tabular}{|c|c|c|c|c|c|c|c|}
\hline \multicolumn{8}{|c|}{ Tensão 500kV } \\
\hline \multirow{2}{*}{$\begin{array}{l}\hat{A} \text { Angulo de } \\
\text { sincronização } \\
\text { real (graus) }\end{array}$} & \multicolumn{6}{|c|}{ Parâmetros } & \multirow{2}{*}{$\begin{array}{c}\text { Ângulo de } \\
\text { sincronização } \\
\text { calculado } \\
\text { (graus) }\end{array}$} \\
\hline & $\begin{array}{c}\beta \\
(\text { graus })\end{array}$ & $\begin{array}{c}\Phi \\
(\text { graus })\end{array}$ & $x$ & $y$ & $\begin{array}{c}I_{R_{1}} \\
(p u)\end{array}$ & $\begin{array}{c}I_{S_{1}} \\
(p u)\end{array}$ & \\
\hline 120 & $-60,1998$ & 0,0357 & - & + & 1,8000 & 1,3000 & $-60,24$ \\
\hline 90 & $-89,8002$ & 0,0357 & - & - & 1,8000 & 1,3000 & 269,76 \\
\hline 60 & $-59,8002$ & 0,0357 & - & - & 1,8000 & 1,3000 & 239,76 \\
\hline 30 & $-29,8002$ & 0,0357 & - & - & 1,8000 & 1,3000 & 209,76 \\
\hline 0 & 0,1998 & 0,0357 & + & - & 1,8000 & 1,3000 & 179,76 \\
\hline-30 & 30,1998 & 0,0357 & + & - & 1,8000 & 1,3000 & 149,76 \\
\hline-60 & 60,1998 & 0,0357 & + & - & 1,8000 & 1,3000 & 119,76 \\
\hline-90 & 89,8002 & 0,0357 & + & + & 1,8000 & 1,3000 & 89,76 \\
\hline-120 & 59,8002 & 0,0357 & + & + & 1,8000 & 1,3000 & 59,76 \\
\hline
\end{tabular}

A partir da análise destes parâmetros, nestas situações de operação do sistema elétrico, elaborou-se o algoritmo proposto neste trabalho, mostrado abaixo.

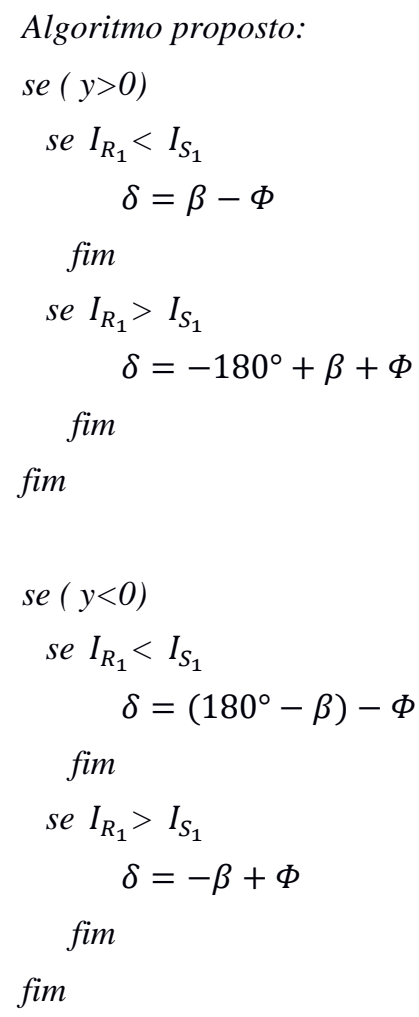

\section{SISTEMA ANALISADO}

Para a análise de desempenho do algoritmo proposto, foram realizadas simulações no software ATPDraw, para sistemas elétricos fictícios e no software ANAREDE para sistemas reais.

A Figura 3 ilustra o sistema elétrico modelado no ATPDraw para os níveis de tensão de 230 e $500 \mathrm{kV}$. A linha de transmissão 2 (LT2), possui $180 \mathrm{~km}$ de comprimento para a tensão de $230 \mathrm{kV}$ e $250 \mathrm{~km}$ para $500 \mathrm{kV}$, sendo em seus terminais onde são coletados os fasores de tensão e corrente. As linhas de transmissão 1 e 3 (LT1 e LT3) tem $20 \mathrm{~km}$ de comprimento nos dois níveis de tensão. Estas simulações foram realizadas considerando 
linhas de transmissão transpostas, utilizando a modelagem de parâmetros distribuídos. Os parâmetros de sequência positiva e zero das linhas são mostrados nas Tabelas 6 e 7.

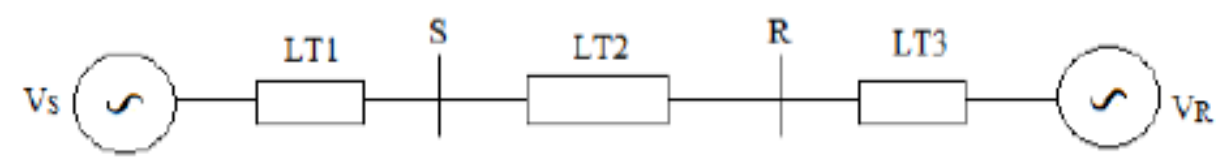

FIGURA 3. Representação unifilar da LT. [2]

TABELA 6. Parâmetros de sequência positiva e zero da linha de 230 kV. (Autoria própria)

\begin{tabular}{cccc}
\hline \multicolumn{4}{c}{ Tensão $230 \mathrm{kV}$} \\
\hline Sequência & $R(\Omega / \mathrm{km})$ & $L(\mathrm{mH} / \mathrm{km})$ & $C(\mu \mathrm{F} / \mathrm{km})$ \\
\hline Positiva & 0,09127 & 1,376 & 0,00847 \\
\hline Zero & 0,44016 & 4,1538 & 0,00610 \\
\hline
\end{tabular}

TABELA 7. Parâmetros de sequência positiva e zero da linha de 500 kV. (Autoria própria)

\begin{tabular}{cccc}
\hline \multicolumn{4}{c}{ Tensão $500 \mathrm{kV}$} \\
\hline Sequência & $R(\Omega / \mathrm{km})$ & $L(\mathrm{mH} / \mathrm{km})$ & $C(\mu F / \mathrm{km})$ \\
\hline Positiva & 0,0221 & 0,852 & 0,01346 \\
\hline Zero & 0,3777 & 3,7767 & 0,00858 \\
\hline
\end{tabular}

Para cada nível de tensão simulado no ATPDraw, foram testadas três situações de operação do sistema elétrico como pode ser visto na Tabela 8. Foi considerado em todos os casos a fase de $V_{S}$ igual a $0^{\circ}$ e $V_{R}$ igual a $-10^{\circ}$. Em todas as simulações foram utilizadas uma taxa de 256 amostras por ciclo.

TABELA 8. Situações analisadas do sistema elétrico. (Autoria própria)

\begin{tabular}{cccc}
\hline \multicolumn{4}{c}{ Situações de operação do sistema elétrico } \\
\hline Parâmetro & \multicolumn{3}{c}{ Tensão $($ pu $)$} \\
\hline $\mathrm{V}_{\mathrm{S}}$ & 1 & 1 & 1 \\
\hline $\mathrm{V}_{\mathrm{R}}$ & 0,95 & 1 & 1,05 \\
\hline
\end{tabular}

No software ANAREDE simulou-se sistemas elétricos reais para 230 e $500 \mathrm{kV}$. Para $230 \mathrm{kV}$ foi simulada a linha de transmissão Natal II (5241) - Natal III (5245) com 11,55 km de comprimento. Já para o nível de tensão de $500 \mathrm{kV}$ foi simulada a linha de Transmissão Luiz Gonzaga (5050) - Milagres (5408) com 231,2 km de comprimento. Na Tabela 9 são mostrados os parâmetros de sequência positiva para as linhas de transmissão de 230 e $500 \mathrm{kV}$.

TABELA 9. Parâmetros de sequência positiva - LTs simuladas no ANAREDE. (Autoria própria)

\begin{tabular}{cccc}
\hline \multicolumn{4}{c}{ Parâmetros sequência positiva } \\
\hline Tensão $(k V)$ & $R(\Omega / \mathrm{km})$ & $L(\mathrm{mH} / \mathrm{km})$ & $C(\mu \mathrm{F} / \mathrm{km})$ \\
\hline 230 & 0,0504 & 0,935 & 0,00866 \\
\hline 500 & 0,0238 & 0,746 & 0,016
\end{tabular}

\section{RESUltAdOS}

Com base nas simulações realizadas nos softwares ATPDraw e ANAREDE, este tópico expõe os resultados avaliativos do algoritmo de sincronização proposto. Entretanto, os valores de tensão oriundos desses softwares são sincronizados automaticamente por eles, sendo necessário à realização de uma dessincronização artificial.

Para uma melhor avaliação do algoritmo de sincronização apontado, foram inseridos erros de sincronização nos fasores de tensão e corrente nos terminais R, iguais aos utilizados em [2] para uma melhor comparação dos dados. 
Na Tabela 10 são expostos os ângulos de sincronização calculados pela metodologia relatada neste artigo, como também pelos algoritmos demonstrados em [1] e [2]. Isto para as linhas de transmissão de 230 e $500 \mathrm{kV}$ simuladas no ATPDraw, para a condição de tensão de 0,95 pu no terminal $\mathrm{R}$.

TABELA 10. Ângulos calculados para situação de operação de 0,95 pu do sistema, para LTs de 230 e 500 $\mathrm{kV}$ (Autoria própria)

\begin{tabular}{|c|c|c|c|c|c|c|}
\hline \multirow{3}{*}{$\begin{array}{l}\text { Ângulo de } \\
\text { sincronização } \\
\text { real (graus) }\end{array}$} & \multicolumn{6}{|c|}{ Ângulo de sincronização calculado (graus) } \\
\hline & \multicolumn{2}{|c|}{ Algoritmo proposto } & \multicolumn{2}{|c|}{$\begin{array}{c}\text { Algoritmo proposto em } \\
{[1]}\end{array}$} & \multicolumn{2}{|c|}{$\begin{array}{c}\text { Algoritmo proposto em } \\
{[2]}\end{array}$} \\
\hline & $230 \mathrm{kV}$ & $500 \mathrm{kV}$ & $230 \mathrm{kV}$ & $500 \mathrm{kV}$ & $230 \mathrm{kV}$ & $500 \mathrm{kV}$ \\
\hline 120 & $-239,72$ & $-239,85$ & $-72,79$ & $-62,77$ & $-239,73$ & $-239,85$ \\
\hline 90 & 90,27 & 90,15 & 257,21 & 267,23 & 90,27 & 90,15 \\
\hline 60 & 60,27 & 60,15 & 227,21 & 237,23 & 60,27 & 60,15 \\
\hline 30 & 30,27 & 30,15 & 197,21 & 207,23 & 30,27 & 30,15 \\
\hline 0 & 0,27 & 0,15 & 167,21 & 177,23 & 0,27 & 0,15 \\
\hline-30 & $-29,73$ & $-29,85$ & 137,21 & 147,23 & $-29,73$ & $-29,85$ \\
\hline-60 & $-59,73$ & $-59,85$ & 107,21 & 117,23 & $-60,73$ & $-59,85$ \\
\hline-90 & $-89,73$ & $-89,85$ & 77,21 & 87,23 & $-89,73$ & $-89,85$ \\
\hline-120 & $-119,73$ & $-119,85$ & 47,21 & 57,23 & $-119,73$ & $-119,85$ \\
\hline
\end{tabular}

Nota-se que o algoritmo mostrado em [1] apresentou valores incorretos para ambos os níveis de tensão. Para o algoritmo deste estudo, foram encontrados valores corretos de ângulos de sincronização, estes bem parecidos com os encontrados pela metodologia realizada em [2].

Os ângulos de sincronização calculados não são exatos devido às margens de erro decorrentes das aproximações numéricas realizadas pelo algoritmo. Contudo, esta margem de erro é bem pequena, sendo que para o algoritmo proposto ela é de $0,27^{\circ}$ para a tensão de $230 \mathrm{kV}$ e de $0,15^{\circ}$ para a tensão de $500 \mathrm{kV}$.

Ainda utilizando os dados das simulações realizadas no ATPDraw, as Tabelas 11 e 12 trazem os ângulos de sincronização calculados para os níveis de 1 pu e 1,05 pu no terminal R.

TABELA 11. Ângulos calculados para situação de operação de 1 pu do sistema, para LTs de 230 e 500 kV (Autoria própria)

\begin{tabular}{|c|c|c|c|c|c|c|}
\hline \multirow{3}{*}{$\begin{array}{c}\hat{A} \text { ngulo de } \\
\text { sincronização } \\
\text { real (graus) }\end{array}$} & \multicolumn{6}{|c|}{ Ângulo de sincronização calculado (graus) } \\
\hline & \multicolumn{2}{|c|}{ Algoritmo proposto } & \multicolumn{2}{|c|}{$\begin{array}{c}\text { Algoritmo proposto em } \\
{[1]}\end{array}$} & \multicolumn{2}{|c|}{$\begin{array}{c}\text { Algoritmo proposto em } \\
{[2]}\end{array}$} \\
\hline & $230 \mathrm{kV}$ & $500 \mathrm{kV}$ & $230 \mathrm{kV}$ & $500 \mathrm{kV}$ & $230 \mathrm{kV}$ & $500 \mathrm{kV}$ \\
\hline 120 & 119,81 & 119,50 & 119,81 & 119,50 & 119,81 & 119,50 \\
\hline 90 & 89,81 & 89,50 & 89,81 & 89,50 & 89,81 & 89,50 \\
\hline 60 & 59,81 & 59,50 & 59,81 & 60,50 & 59,81 & 60,50 \\
\hline 30 & 29,81 & 29,50 & 29,81 & 29,50 & 29,81 & 29,50 \\
\hline 0 & $-0,19$ & $-0,50$ & $-0,19$ & 0,50 & $-0,19$ & 0,50 \\
\hline-30 & $-30,19$ & $-30,50$ & $-30,19$ & $-30,50$ & $-30,19$ & $-30,50$ \\
\hline-60 & $-60,19$ & $-60,50$ & $-60,19$ & $-60,50$ & $-60,19$ & $-60,50$ \\
\hline-90 & $-90,19$ & $-90,50$ & $-90,19$ & $-90,50$ & $-90,19$ & $-90,50$ \\
\hline-120 & 239,81 & 239,50 & 239,81 & 239,50 & 239,81 & 239,50 \\
\hline
\end{tabular}


TABELA 12. Ângulos calculados para situação de operação de 1,05 pu do sistema, para LTs de 230 e 500 $\mathrm{kV}$ (Autoria própria)

\begin{tabular}{ccccccc}
\hline \multirow{2}{*}{$\begin{array}{c}\text { Ângulo de } \\
\text { sincronização } \\
\text { real }(\text { graus) }\end{array}$} & Algoritmo proposto & Algoritmo proposto em & Algoritmo proposto em \\
\cline { 2 - 7 } & $230 \mathrm{kV}$ & $500 \mathrm{kV}$ & $230 \mathrm{kV}$ & $500 \mathrm{kV}$ & $230 \mathrm{kV}$ & $500 \mathrm{kV}$ \\
\hline 120 & 119,93 & 119,90 & 119,95 & 119,90 & 119,95 & 119,90 \\
\hline 90 & 89,93 & 89,90 & 89,95 & 89,90 & 89,95 & 89,90 \\
\hline 60 & 59,93 & 59,90 & 59,95 & 59,90 & 59,95 & 59,90 \\
\hline 30 & 29,93 & 29,90 & 29,95 & 29,90 & 29,95 & 29,90 \\
\hline 0 & $-0,07$ & $-0,10$ & 0,045 & 0,10 & 0,045 & 0,10 \\
\hline-30 & $-30,07$ & $-30,10$ & $-30,05$ & $-30,10$ & $-30,05$ & $-30,10$ \\
\hline-60 & $-60,07$ & $-60,10$ & $-60,05$ & $-60,10$ & $-60,05$ & $-60,10$ \\
\hline-90 & $-90,07$ & $-90,10$ & $-90,05$ & $-90,10$ & $-90,05$ & $-90,10$ \\
\hline-120 & 239,93 & 239,90 & 239,95 & 239,90 & 239,95 & 239,90 \\
\hline
\end{tabular}

Para estas condições, o algoritmo proposto neste trabalho, como também os colocados em [1] e em [2] apresentaram valores de ângulos de sincronização aproximados. Para a condição de 1 pu, foram encontrados erros absolutos de $0,19^{\circ}$ para a tensão de $230 \mathrm{kV}$ e de $0,5^{\circ}$ para $500 \mathrm{kV}$. Já o erro absoluto encontrado para a condição de 1,05 pu foi de $0,07^{\circ}$ para a tensão de $230 \mathrm{kV}$ e de $0,1^{\circ}$ para a tensão de $500 \mathrm{kV}$.

$\mathrm{Na}$ Tabela 13 podem-se observar os valores dos ângulos de sincronização calculados pelo algoritmo desenvolvido nesse estudo, e também em [1] e [2], para os casos de sistemas reais simulados no ANAREDE com linhas de transmissão de 230 e 500 kV.

TABELA 13. Ângulos calculados para sistemas reais simulados no ANAREDE, para LTs de 230 e $500 \mathrm{kV}$ (Autoria própria)

\begin{tabular}{|c|c|c|c|c|c|c|}
\hline \multirow{3}{*}{$\begin{array}{l}\hat{A} \text { Angulo de } \\
\text { sincronização } \\
\text { real (graus) }\end{array}$} & \multicolumn{6}{|c|}{ Ângulo de sincronização calculado (graus) } \\
\hline & \multicolumn{2}{|c|}{ Algoritmo proposto } & \multicolumn{2}{|c|}{$\begin{array}{c}\text { Algoritmo proposto } \\
\text { em }[1]\end{array}$} & \multicolumn{2}{|c|}{$\begin{array}{c}\text { Algoritmo proposto } \\
\text { em [2] }\end{array}$} \\
\hline & $230 \mathrm{kV}$ & $500 \mathrm{kV}$ & $230 k \mathrm{~V}$ & $500 \mathrm{kV}$ & $230 \mathrm{kV}$ & $500 \mathrm{kV}$ \\
\hline 120 & 120,13 & 119,84 & 120,04 & $-60,24$ & 120,04 & $-240,17$ \\
\hline 90 & 90,13 & 89,85 & 90,04 & 269,76 & 90,04 & 89,83 \\
\hline 60 & 60,13 & 59,85 & 60,04 & 239,76 & 60,04 & 59,83 \\
\hline 30 & 30,13 & 29,85 & 30,04 & 209,76 & 30,04 & 29,83 \\
\hline 0 & 0,13 & $-0,16$ & 0,04 & 179,76 & 0,04 & 0,17 \\
\hline-30 & $-29,87$ & $-30,16$ & $-29,96$ & 149,76 & $-29,96$ & $-30,17$ \\
\hline-60 & $-59,87$ & $-60,16$ & $-59,96$ & 119,76 & $-59,96$ & $-60,17$ \\
\hline-90 & $-89,87$ & 269,84 & $-89,96$ & 89,76 & $-89,96$ & $-90,17$ \\
\hline-120 & 240,13 & 239,84 & 240,04 & 59,76 & 240,04 & $-120,17$ \\
\hline
\end{tabular}

É notório que para a tensão de $230 \mathrm{kV}$, todos os algoritmos sugeridos apresentaram ângulos de sincronização corretos, cada um com sua margem de erro respectiva. Todavia, para tensão de $500 \mathrm{kV}$, o código proposto em [1] apresentou valores imprecisos, enquanto que o código aprimorado neste trabalho e o desenvolvido por [2] obtiveram valores de ângulos de sincronização mais precisos. A metodologia proposta nesse artigo apontou erro absoluto de apenas $0,13^{\circ}$ para a tensão de $230 \mathrm{kV}$ e de $0,16^{\circ}$ para tensão de $500 \mathrm{kV}$.

\section{CONCLUSÃO}

R4EM, v.1, n.1, p. 100-111, 2019 
A partir do estudo realizado neste trabalho foi possível obter um novo equacionamento para a sincronização de registros, sendo uma versão aperfeiçoada das metodologias propostas em [1] e [2]. Ou seja, conseguiu-se eliminar as limitações do algoritmo proposto em [1], fundamentando-se no uso das condições das correntes terminais, proposta em [2]. Com isso, obteve-se uma eficiente ferramenta de sincronização que funciona com um número reduzido de equações, para qualquer situação do sistema elétrico. Além disso, preserva a característica de ser independente dos parâmetros elétricos da linha, sendo dependente unicamente dos fasores de tensão e corrente pré-falta.

\section{REFERÊNCIAS}

[1] CRUZ, Melinda Cesianara Silva da; OLIVEIRA, José Tavares de; MEDEIROS JUNIOR, Manoel Firmino de. Sincronização de Registros Oscilográficos em Linhas de Transmissão com Parâmetros Elétricos Desconhecidos. In: SIMPÓSIO BRASILEIRO DE SISTEMAS ELÉTRICOS, 6., 2016, Natal. Anais... . Natal: Simpósio Brasileiro de Sistemas Elétricos, 2016. p. 1 - 4.

[2] MONTEIRO, Roana D. S. et al. Um novo algoritmo para sincronização de Registros Oscilográficos em Linhas de Transmissão com Parâmetros Elétricos Desconhecidos. XXII Congresso Brasileiro de Automática, João Pessoa, 2018. p. $1-7$.

[3] CRUZ, M. C. S. da, Almeida, M. A. D., Oliveira, J. T., Cândido, C. K. S. S., Leitão, J. J. A. L., Medeiros Jr, M. F. (2010). Localização de Faltas em Linhas de Transmissão de Múltiplos Terminais a Partir de Registros Oscilográficos Sincronizados Via Transformada Wavelet. In: IEEE/PES Transmission \& Distribution Conference and Exposition Latin America.

[4] COURY, Denis Vinicius; OLESKOVICZ, Mário; GIOVANINI, Renan. Proteção Digital de Sistemas Elétricos de Potência: dos relés eletromecânicos aos microprocessados inteligentes. São Carlos-sp: Eesc-usp, 2007. $378 \mathrm{p}$.

[5] CRUZ, Melinda Cesianara Souza et al. Metodologia para Sincronização de Registros de Falta baseada em Equações de Circuito Equivalente de Linhas de Transmissão. Ieee/ias International Conference On Industry Applications, Fortaleza, v. 10, n. 10, p.120-126, fev. 2012

[6] Y. Liao e N. Kang, "Fault-Location Algorithms Without utilizing Line Parameters Based on the Distributed Parameter Line Model”, IEEE Trans. Power Deliv., vol. 24, no. 2, p. 579-584, 2009. 